Supporting Information

\title{
Methanesulfonyl Azide: Molecular Structure and Photolysis in Solid Noble Gas Matrices
}

\author{
Guohai Deng, ${ }^{\dagger}$ Dingqing $L i,^{\dagger}$ Zhuang $W u,,^{\dagger}$ Hongmin $\mathrm{Li}^{\dagger}$ \\ Eduard Bernhardt, ${ }^{*}$ and Xiaoqing Zeng, ${ }^{\dagger, *}$ \\ $†$ College of Chemistry, Chemical Engineering and Materials Science, Soochow University, \\ 215123 Suzhou, China \\ E-mail: xqzeng@suda.edu.cn; Phone: +86 51265883583
}

†FB C-Anorganische Chemie, Bergische Universität Wuppertal, Gaussstrasse 20, 42119

Wuppertal, Germany 
Table S1. Calculated IR frequencies $\left(\mathrm{cm}^{-1}\right)$ and intensities $\left(\mathrm{km} \mathrm{mol}^{-1}\right.$, in paretheses) for $\mathrm{CH}_{3} \mathrm{SO}_{2} \mathrm{~N}$ isomers with different theoretical methods.

\begin{tabular}{|c|c|c|c|c|c|c|c|c|}
\hline \multicolumn{4}{|c|}{$\mathrm{CH}_{3} \mathrm{SO}_{2} \mathrm{~N}\left({ }^{1} \mathrm{~A}\right)$} & \multicolumn{5}{|c|}{$\mathrm{CH}_{3} \mathrm{SO}_{2} \mathrm{~N}\left({ }^{3} \mathrm{~A}\right)$} \\
\hline $\mathrm{B} 3 \mathrm{LYP}^{\mathrm{a}}$ & M06-2X & MPW1PW91 $^{\mathrm{a}}$ & CBS-QB3 & B3LYP $^{a}$ & M06-2X & MPW1PW91 $^{\mathrm{a}}$ & CBS-QB3 & $\mathrm{B} 3 \mathrm{LYP}^{\mathrm{b}}$ \\
\hline$v$ & $v$ & $v$ & $v$ & $v$ & $v$ & $v$ & $v$ & $\Delta v\left({ }^{14 / 15} \mathrm{~N}\right)$ \\
\hline $3183(<1)$ & $3206(3)$ & $3213(1)$ & $3182(<1)$ & $3170(<1)$ & $3185(2)$ & $3201(<1)$ & $3169(<1)$ & 0 \\
\hline $3149(<1)$ & $3179(2)$ & $3179(<1)$ & $3148(<1)$ & $3147(2)$ & $3170(4)$ & $3179(3)$ & $3146(1)$ & 0 \\
\hline $3061(<1)$ & $3081(1)$ & $3084(<1)$ & $3059(<1)$ & $3056(2)$ & $3068(3)$ & $3078(3)$ & $3053(1)$ & 0 \\
\hline $1456(1)$ & $1463(14)$ & $1455(7)$ & 1457(4) & 1452(9) & $1454(7)$ & 1449(9) & $1453(11)$ & 0 \\
\hline 1451(9) & $1453(9)$ & $1447(10)$ & $1452(11)$ & $1447(4)$ & $1453(5)$ & $1444(<1)$ & $1448(7)$ & 0 \\
\hline 1382(207) & $1434(213)$ & $1422(208)$ & $1355(117)$ & $1349(14)$ & $1424(232)$ & $1390(206)$ & $1344(15)$ & 0 \\
\hline 1353(9) & $1364(10)$ & $1353(11)$ & $1346(81)$ & 1341(187) & $1354(25)$ & $1348(23)$ & $1317(168)$ & 0 \\
\hline $1059(76)$ & $1109(75)$ & 1096(75) & $1027(80)$ & 1154(97) & $1222(123)$ & 1194(101) & $1125(101)$ & 0 \\
\hline 994(15) & 1014(23) & $1014(21)$ & $986(6)$ & $971(2)$ & $973(2)$ & $967(2)$ & $969(2)$ & 0 \\
\hline $984(6)$ & 994(4) & $983(4)$ & 976(14) & $965(17)$ & $972(30)$ & $965(22)$ & $960(18)$ & 0 \\
\hline $935(4)$ & $954(4)$ & $946(4)$ & $909(9)$ & $741(46)$ & 787(47) & $771(46)$ & $723(52)$ & 3.0 \\
\hline $718(30)$ & $770(30)$ & $751(30)$ & $705(32)$ & $658(3)$ & $718(4)$ & $693(2)$ & 631(1) & 11.0 \\
\hline $478(48)$ & $530(36)$ & 494(48) & $469(48)$ & $479(30)$ & $501(40)$ & 492(34) & $469(31)$ & 3.0 \\
\hline $434(16)$ & $472(35)$ & $454(17)$ & $414(17)$ & $446(38)$ & $464(41)$ & 457(39) & $435(37)$ & 1.0 \\
\hline $386(5)$ & $418(11)$ & $405(7)$ & $360(5)$ & $342(2)$ & $355(4)$ & $348(3)$ & $336(3)$ & 4.0 \\
\hline $325(<1)$ & $334(<1)$ & $329(<1)$ & $319(1)$ & $273(3)$ & $290(3)$ & $281(3)$ & $266(4)$ & 4.0 \\
\hline $270(<1)$ & $290(<1)$ & $276(<1)$ & $261(<1)$ & $261(3)$ & $272(2)$ & $266(3)$ & $260(3)$ & 0 \\
\hline $171(<1)$ & $171(<1)$ & $174(<1)$ & $156(<1)$ & $181(<1)$ & $182(<1)$ & $188(<1)$ & $162(<1)$ & 0 \\
\hline
\end{tabular}

${ }^{a}$ The $6-311++\mathrm{G}(3 \mathrm{df}, 3 \mathrm{pd})$ basis set was used. ${ }^{b}$ The calculated isotope shift $\left(\mathrm{cm}^{-1}\right)$ with ${ }^{15} \mathrm{~N}$ labeling. 
Table S1. continued.

\begin{tabular}{|c|c|c|c|c|}
\hline \multicolumn{5}{|c|}{$\mathrm{CH}_{3} \mathrm{NSO}_{2}$} \\
\hline B3LYP ${ }^{a}$ & M06- $2 X^{\mathrm{a}}$ & MPW1PW91 ${ }^{\mathrm{a}}$ & CBS-QB3 & B3LYP ${ }^{b}$ \\
\hline$v$ & $v$ & $v$ & $v$ & $\Delta v\left(\left(^{14 / 15} \mathrm{~N}\right)\right.$ \\
\hline $3118(5)$ & $3149(3)$ & $3147(4)$ & $3115(7)$ & 0 \\
\hline $3108(7)$ & $3148(2)$ & $3140(6)$ & $3105(11)$ & 0 \\
\hline $3039(26)$ & $3065(23)$ & $3061(26)$ & $3035(27)$ & 0 \\
\hline $1497(14)$ & $1512(21)$ & $1498(17)$ & $1496(14)$ & 0 \\
\hline $1492(8)$ & $1504(8)$ & 1491(8) & 1491(8) & 0 \\
\hline 1461(3) & $1475(30)$ & $1464(12)$ & $1457(<1)$ & 0 \\
\hline $1375(215)$ & $1442(250)$ & $1420(222)$ & $1345(203)$ & 2.0 \\
\hline $1321(222)$ & $1390(222)$ & $1364(218)$ & $1297(207)$ & 16.9 \\
\hline $1157(18)$ & 1191(41) & $1169(33)$ & $1154(20)$ & 3.0 \\
\hline $1129(<1)$ & $1160(10)$ & $1147(14)$ & $1125(<1)$ & 2.0 \\
\hline $1116(27)$ & $1139(<1)$ & $1131(<1)$ & 1093(35) & 12.4 \\
\hline $835(16)$ & $889(20)$ & $868(17)$ & $827(16)$ & 9.0 \\
\hline $550(10)$ & $569(13)$ & $559(11)$ & $545(9)$ & 5.0 \\
\hline $487(26)$ & $506(30)$ & $497(27)$ & $479(29)$ & 1.0 \\
\hline $427(23)$ & $462(30)$ & $445(24)$ & $427(21)$ & 2.0 \\
\hline 233(7) & $244(7)$ & $236(7)$ & $242(7)$ & 0 \\
\hline $214(1)$ & $218(1)$ & $218(1)$ & $212(2)$ & 3.0 \\
\hline $86(<1)$ & $145(<1)$ & $95(<1)$ & $94(<1)$ & 0 \\
\hline
\end{tabular}


Table S1. continued.

\begin{tabular}{|c|c|c|c|c|}
\hline \multicolumn{5}{|c|}{ syn- $\mathrm{CH}_{3} \mathrm{~S}(\mathrm{O}) \mathrm{NO}$} \\
\hline B3LYP & M06-2X & MPW1PW91 & CBS-QB3 & B3LYP \\
\hline$v$ & $v$ & $v$ & $v$ & $\Delta v\left(\left({ }^{14 / 15} \mathrm{~N}\right)\right.$ \\
\hline $3141(<1)$ & $3166(<1)$ & $3174(<1)$ & $3137(1)$ & 0 \\
\hline $3136(2)$ & $3162(<1)$ & $3169(<1)$ & $3134(4)$ & 0 \\
\hline $3039(3)$ & $3056(<1)$ & $3063(2)$ & $3035(5)$ & 0 \\
\hline 1804(504) & $1830(427)$ & $1826(445)$ & $1831(524)$ & 32.0 \\
\hline $1466(9)$ & $1470(9)$ & $1463(9)$ & $1467(11)$ & 0 \\
\hline $1444(7)$ & $1447(9)$ & $1440(8)$ & $1443(7)$ & 0 \\
\hline $1320(8)$ & $1327(8)$ & $1320(9)$ & $1312(9)$ & 0 \\
\hline $1135(157)$ & $1198(171)$ & $1177(158)$ & $1098(138)$ & 0 \\
\hline $944(8)$ & $959(10)$ & $947(9)$ & $935(11)$ & 0 \\
\hline $926(2)$ & $936(<1)$ & 931(2) & $918(2)$ & 0 \\
\hline $655(18)$ & $703(21)$ & $690(19)$ & $642(20)$ & 0 \\
\hline $604(13)$ & $651(21)$ & $636(17)$ & $599(11)$ & 11.0 \\
\hline $350(13)$ & $371(16)$ & $358(14)$ & $343(13)$ & 0 \\
\hline $299(21)$ & $346(28)$ & $316(27)$ & $310(15)$ & 3.0 \\
\hline $233(2)$ & $276(<1)$ & $253(2)$ & $226(1)$ & 4.0 \\
\hline $179(<1)$ & $211(<1)$ & $189(<1)$ & $174(<1)$ & 0 \\
\hline $142(<1)$ & $178(<1)$ & $156(<1)$ & $143(<1)$ & 0 \\
\hline $136(<1)$ & $142(1)$ & $140(1)$ & $130(<1)$ & 0 \\
\hline
\end{tabular}


Table S1. continued.

\begin{tabular}{ccccc}
\hline & \multicolumn{3}{c}{ CH2NSO2H } & \\
\hline B3LYP & M06-2X & MPW1PW91 & CBS-QB3 & B3LYP \\
$v$ & $v$ & $v$ & $v$ & $\Delta v\left({ }^{14 / 15} \mathrm{~N}\right)$ \\
\hline $3166(2)$ & $3201(<1)$ & $3194(2)$ & $3159(4)$ & 0 \\
$3062(16)$ & $3101(14)$ & $3084(16)$ & $3054(16)$ & 0 \\
$2563(32)$ & $2649(18)$ & $2603(29)$ & $2544(45)$ & 0 \\
$1696(54)$ & $1736(69)$ & $1723(61)$ & $1690(50)$ & 21.0 \\
$1479(26)$ & $1498(23)$ & $1480(23)$ & $1473(22)$ & 8.0 \\
$1384(250)$ & $1434(271)$ & $1420(257)$ & $1364(232)$ & 0 \\
$1216(42)$ & $1241(110)$ & $1226(105)$ & $1212(24)$ & 4.0 \\
$1175(123)$ & $1214(67)$ & $1200(60)$ & $1153(146)$ & 0 \\
$1129(14)$ & $1152(14)$ & $1138(15)$ & $1121(14)$ & 1.0 \\
$1107(6)$ & $1135(5)$ & $1115(8)$ & $1103(3)$ & 0 \\
$1091(9)$ & $1110(20)$ & $1102(11)$ & $1082(7)$ & 0 \\
$742(61)$ & $762(90)$ & $756(79)$ & $740(49)$ & 4.0 \\
$644(72)$ & $688(49)$ & $676(53)$ & $620(88)$ & 7.0 \\
$581(24)$ & $598(29)$ & $589(26)$ & $575(23)$ & 5.0 \\
$488(16)$ & $508(21)$ & $499(18)$ & $480(17)$ & 3.0 \\
$382(17)$ & $409(22)$ & $394(18)$ & $373(18)$ & 2.0 \\
$286(15)$ & $317(14)$ & $293(16)$ & $289(14)$ & 1.0 \\
$96(12)$ & $115(12)$ & $102(12)$ & $102(11)$ & 1.0 \\
\hline & & & & \\
\hline
\end{tabular}


Table S1. continued.

\begin{tabular}{ccccc}
\hline \multicolumn{5}{c}{ anti-CH3S(O)NO } \\
\hline B3LYP & M06-2X & MPW1PW91 & CBS-QB3 & B3LYP \\
$v$ & $v$ & $v$ & $v$ & $\Delta v\left({ }^{14 / 15} \mathrm{~N}\right)$ \\
\hline $3140(<1)$ & $3167(<1)$ & $3174(<1)$ & $3137(2)$ & 0 \\
$3136(1)$ & $3161(<1)$ & $3168(<1)$ & $3133(2)$ & 0 \\
$3037(2)$ & $3053(<1)$ & $3061(1)$ & $3033(4)$ & 0 \\
$1760(527)$ & $1787(373)$ & $1786(447)$ & $1778(553)$ & 31.0 \\
$1464(9)$ & $1470(9)$ & $1462(10)$ & $1466(11)$ & 0 \\
$1445(5)$ & $1449(6)$ & $1442(6)$ & $1446(5)$ & 0 \\
$1319(10)$ & $1322(13)$ & $1318(13)$ & $1315(12)$ & 0 \\
$1150(213)$ & $1208(220)$ & $1190(212)$ & $1118(191)$ & 0 \\
$948(9)$ & $957(10)$ & $950(9)$ & $942(13)$ & 0 \\
$925(2)$ & $938(3)$ & $928(2)$ & $920(2)$ & 0 \\
$646(12)$ & $703(9)$ & $681(10)$ & $633(14)$ & 0 \\
$621(17)$ & $669(21)$ & $646(21)$ & $609(14)$ & 11.0 \\
$338(8)$ & $357(10)$ & $345(9)$ & $327(8)$ & 0 \\
$253(9)$ & $313(14)$ & $274(13)$ & $249(7)$ & 1.0 \\
$225(8)$ & $276(2)$ & $247(5)$ & $220(7)$ & 2.0 \\
$189(3)$ & $211(8)$ & $197(3)$ & $172(3)$ & 0 \\
$175(5)$ & $204(2)$ & $188(5)$ & $158(6)$ & 0 \\
$67(<1)$ & $98(1)$ & $76(<1)$ & $60(<1)$ & 0 \\
\hline & & & &
\end{tabular}


Table S2. Calculated structural parameters for $\mathrm{CH}_{3} \mathrm{SO}_{2} \mathrm{~N}$ isomers with different theoretical methods. Bond lengths and angles are given in $[\AA]$ and $\left[^{\circ}\right]$, respectively.

\begin{tabular}{|c|c|c|c|c|c|c|c|c|}
\hline \multirow{2}{*}{ parameter } & \multicolumn{4}{|c|}{$\mathrm{CH}_{3} \mathrm{SO}_{2} \mathrm{~N}\left({ }^{1} \mathrm{~A}\right)$} & \multicolumn{4}{|c|}{$\mathrm{CH}_{3} \mathrm{SO}_{2} \mathrm{~N}\left({ }^{3} \mathrm{~A}\right)$} \\
\hline & B3LYP & M06-2X & MPW1PW91 & CBS-QB3 & B3LYP & M06-2X & MPW1PW91 & CBS-QB3 \\
\hline$r(\mathrm{~S}-\mathrm{O} 1)$ & 1.520 & 1.516 & 1.514 & 1.530 & 1.438 & 1.427 & 1.430 & 1.444 \\
\hline$r(\mathrm{~S}-\mathrm{O} 2)$ & 1.428 & 1.419 & 1.421 & 1.434 & 1.438 & 1.427 & 1.430 & 1.444 \\
\hline$r(\mathrm{~S}-\mathrm{C})$ & 1.776 & 1.761 & 1.759 & 1.785 & 1.778 & 1.764 & 1.762 & 1.787 \\
\hline$r(\mathrm{~S}-\mathrm{N})$ & 1.551 & 1.542 & 1.542 & 1.557 & 1.689 & 1.696 & 1.683 & 1.699 \\
\hline$\angle(\mathrm{O} 1 \mathrm{SO} 2)$ & 122.8 & 123.0 & 123.0 & 123.2 & 120.8 & 121.4 & 121.0 & 121.2 \\
\hline$\angle(\mathrm{O} 1 \mathrm{SN})$ & 70.7 & 67.5 & 69.0 & 71.9 & 106.6 & 106.6 & 106.6 & 106.7 \\
\hline$\angle(\mathrm{O} 2 \mathrm{SN})$ & 126.8 & 127.8 & 127.3 & 126.7 & 106.6 & 106.6 & 106.6 & 106.7 \\
\hline$\angle(\mathrm{CSN})$ & 115.6 & 116.0 & 115.8 & 115.3 & 101.8 & 100.8 & 101.4 & 101.3 \\
\hline \multirow{2}{*}{ parameter } & \multicolumn{4}{|c|}{$\mathrm{CH}_{3} \mathrm{NSO}_{2}$} & \multicolumn{4}{|c|}{$\mathrm{CH}_{2} \mathrm{NSO}_{2} \mathrm{H}$} \\
\hline & B3LYP & M06-2X & MPW1PW91 & CBS-QB3 & B3LYP & M06-2X & MPW1PW91 & CBS-QB3 \\
\hline$r(\mathrm{~S}-\mathrm{O} 1)$ & 1.438 & 1.426 & 1.429 & 1.446 & 1.437 & 1.428 & 1.430 & 1.443 \\
\hline$r(\mathrm{~S}-\mathrm{O} 2)$ & 1.429 & 1.419 & 1.422 & 1.437 & 1.430 & 1.422 & 1.423 & 1.437 \\
\hline$r(\mathrm{~S}-\mathrm{N})$ & 1.499 & 1.486 & 1.491 & 1.506 & 1.698 & 1.683 & 1.680 & 1.716 \\
\hline$r(\mathrm{C}-\mathrm{N})$ & 1.465 & 1.463 & 1.456 & 1.470 & 1.266 & 1.263 & 1.263 & 1.270 \\
\hline$\angle(\mathrm{O} 1 \mathrm{SO} 2)$ & 120.0 & 119.9 & 120.0 & 120.3 & 121.3 & 121.1 & 121.2 & 121.8 \\
\hline$\angle(\mathrm{O} 1 \mathrm{SN})$ & 122.9 & 122.5 & 122.8 & 122.8 & 107.2 & 107.2 & 107.2 & 107.2 \\
\hline$\angle(\mathrm{O} 2 \mathrm{SN})$ & 117.0 & 117.7 & 117.1 & 116.8 & 112.3 & 111.7 & 112.2 & 121.1 \\
\hline$\angle(\mathrm{CNS})$ & 121.0 & 120.4 & 120.6 & 120.0 & 116.5 & 115.7 & 116.0 & 115.5 \\
\hline \multirow{2}{*}{ parameter } & \multicolumn{4}{|c|}{ syn- $\mathrm{CH}_{3} \mathrm{~S}(\mathrm{O}) \mathrm{NO}$} & \multicolumn{4}{|c|}{ anti- $\mathrm{CH}_{3} \mathrm{~S}(\mathrm{O}) \mathrm{NO}$} \\
\hline & B3LYP & M06-2X & MPW1PW91 & CBS-QB3 & B3LYP & M06-2X & MPW1PW91 & CBS-QB3 \\
\hline$r(\mathrm{~S}-\mathrm{O} 1)$ & 1.480 & 1.467 & 1.470 & 1.490 & 1.476 & 1.464 & 1.467 & 1.485 \\
\hline$r(\mathrm{~N}-\mathrm{O} 2)$ & 1.152 & 1.155 & 1.152 & 1.153 & 1.161 & 1.164 & 1.160 & 1.162 \\
\hline$r(\mathrm{~S}-\mathrm{N})$ & 2.080 & 1.949 & 2.002 & 2.134 & 2.048 & 1.915 & 1.976 & 2.097 \\
\hline$r(\mathrm{C}-\mathrm{S})$ & 1.820 & 1.801 & 1.802 & 1.832 & 1.819 & 1.802 & 1.801 & 1.831 \\
\hline$\angle(\mathrm{O} 1 \mathrm{SN})$ & 100.8 & 103.4 & 102.5 & 97.8 & 105.5 & 105.4 & 105.7 & 107.5 \\
\hline$\angle(\mathrm{O} 2 \mathrm{NS})$ & 106.4 & 107.9 & 107.3 & 103.9 & 113.5 & 112.2 & 113.1 & 111.8 \\
\hline$\angle(\mathrm{CSN})$ & 88.7 & 88.2 & 88.5 & 88.6 & 88.4 & 89.6 & 88.6 & 87.8 \\
\hline
\end{tabular}


Calculated absolute energies (in Hartrees), and the atomic coordinates (in angstroms) for all optimized structures.

\section{$\mathrm{CH}_{3} \mathrm{SO}_{2} \mathrm{~N}_{3}$}

\section{B3LYP/6-311++G(3df,3pd)}

Zero-point correction $=0.060826$ (Hartree/Particle)

Thermal correction to Energy $=0.068061$

Thermal correction to Enthalpy $=0.069005$

Thermal correction to Gibbs Free Energy $=0.028417$

Sum of electronic and zero-point Energies $=-752.819550$

Sum of electronic and thermal Energies $=-752.812316$

Sum of electronic and thermal Enthalpies $=-752.811371$

Sum of electronic and thermal Free Energies $=-752.851960$

$\begin{array}{lccc}\mathrm{C} & -1.37736200 & 1.44200700 & -0.00757800 \\ \mathrm{H} & -1.53771200 & 1.69862400 & -1.05030000 \\ \mathrm{H} & -2.32476200 & 1.38437300 & 0.52433900 \\ \mathrm{H} & -0.70704600 & 2.14376500 & 0.47989100 \\ \mathrm{~S} & -0.66830200 & -0.18698500 & 0.06879300 \\ \mathrm{O} & -0.25792100 & -0.46729200 & 1.41464900 \\ \mathrm{O} & -1.50022300 & -1.08337700 & -0.66784800 \\ \mathrm{~N} & 0.73980300 & 0.03207300 & -0.90698600 \\ \mathrm{~N} & 1.80728900 & 0.07335700 & -0.28243800 \\ \mathrm{~N} & 2.82314700 & 0.11147100 & 0.19177300\end{array}$

\section{CBS-QB3}

Temperature $=298.150000$

$\mathrm{E}(\mathrm{ZPE})=0.059717$

$\mathrm{E}(\mathrm{SCF})=-750.232779$

$\mathrm{DE}(\mathrm{CBS})=-0.143282$

$\mathrm{DE}(\mathrm{CCSD})=-0.042629$

$\mathrm{DE}($ Empirical $)=-0.063151$

CBS-QB3 $(0 \mathrm{~K})=-751.824825$

CBS-QB3 Enthalpy= -751.816451

$\begin{array}{lr}\mathrm{C} & -1.51155800 \\ \mathrm{H} & -1.65417600 \\ \mathrm{H} & -2.47122300 \\ \mathrm{H} & -0.91684700 \\ \mathrm{~S} & -0.66114400 \\ \mathrm{O} & -0.22395200 \\ \mathrm{O} & -1.40501700 \\ \mathrm{~N} & 0.73931400 \\ \mathrm{~N} & 1.81396000 \\ \mathrm{~N} & 2.83553300\end{array}$

Pressure $=1.000000$

$\mathrm{E}($ Thermal $)=0.067147$

$\mathrm{DE}(\mathrm{MP} 2)=-1.436192$

$\mathrm{DE}(\mathrm{MP} 34)=-0.012340$

$\mathrm{DE}(\mathrm{Int})=0.045830$

CBS-QB3 Energy $=-751.817395$

CBS-QB3 Free Energy= -751.857656
1.36715900
0.11165100
1.71322100
$-0.91035400$
1.17555900
0.59302800
2.06741100
0.69502400
$-0.20198700$
0.05452300
$-0.56883300$
1.37761100
$-1.09879800$
$-0.78143900$
0.27794200
$-0.87850400$
0.15017600
$-0.27240700$
$0.05955200 \quad 0.19528900$

\section{M06-2X}

Zero-point correction $=0.062324$ (Hartree/Particle)

Thermal correction to Energy $=0.069289$ 
Thermal correction to Enthalpy $=0.070233$

Thermal correction to Gibbs Free Energy $=0.030434$

Sum of electronic and zero-point Energies $=-752.643729$

Sum of electronic and thermal Energies $=-752.636765$

Sum of electronic and thermal Enthalpies $=-752.635821$

Sum of electronic and thermal Free Energies $=-752.675620$

$\begin{array}{lrrr}\mathrm{C} & -1.34440000 & 1.43813100 & -0.04216500 \\ \mathrm{H} & -2.26598100 & 1.41445700 & 0.53442900 \\ \mathrm{H} & -0.64121000 & 2.14789300 & 0.38236700 \\ \mathrm{H} & -1.54675400 & 1.64586800 & -1.08791100 \\ \mathrm{~S} & -0.65387100 & -0.18080400 & 0.07350900 \\ \mathrm{O} & -0.19866900 & -0.40527200 & 1.40832500 \\ \mathrm{O} & -1.51933200 & -1.09320800 & -0.58593400 \\ \mathrm{~N} & 0.70937100 & -0.02756500 & -0.93682800 \\ \mathrm{~N} & 1.77349000 & 0.05417000 & -0.30413100 \\ \mathrm{~N} & 2.76375200 & 0.12249500 & 0.19365000\end{array}$

\section{MPW1PW91}

Zero-point correction $=0.061740$ (Hartree/Particle)

Thermal correction to Energy $=0.068855$

Thermal correction to Enthalpy $=0.069799$

Thermal correction to Gibbs Free Energy $=0.029531$

Sum of electronic and zero-point Energies $=-752.729055$

Sum of electronic and thermal Energies $=-752.721939$

Sum of electronic and thermal Enthalpies $=-752.720995$

Sum of electronic and thermal Free Energies $=-752.761264$

$\begin{array}{lrrr}\mathrm{C} & -1.31953000 & 1.45137900 & -0.04174200 \\ \mathrm{H} & -1.48674500 & 1.68356300 & -1.08896300 \\ \mathrm{H} & -2.26126700 & 1.43779900 & 0.50280900 \\ \mathrm{H} & -0.62438100 & 2.14769400 & 0.41784400 \\ \mathrm{~S} & -0.66391400 & -0.18017100 & 0.07254400 \\ \mathrm{O} & -0.25239600 & -0.43143000 & 1.41634500 \\ \mathrm{O} & -1.52546200 & -1.06758900 & -0.62623400 \\ \mathrm{~N} & 0.72436300 & -0.04140200 & -0.90689900 \\ \mathrm{~N} & 1.78576800 & 0.04757800 & -0.28766300 \\ \mathrm{~N} & 2.79487700 & 0.12204500 & 0.18558600\end{array}$

\section{$\mathrm{CH}_{3} \mathrm{NSO}_{2}$}

B3LYP/6-311++G(3df,3pd)

Zero-point correction $=0.051596$ (Hartree/Particle)

Thermal correction to Energy $=0.057114$

Thermal correction to Enthalpy $=0.058059$

Thermal correction to Gibbs Free Energy $=0.022283$

Sum of electronic and zero-point Energies $=-643.308631$

Sum of electronic and thermal Energies $=-643.303113$

Sum of electronic and thermal Enthalpies $=-643.302169$

Sum of electronic and thermal Free Energies $=-643.337944$ 


$\begin{array}{lrrr}\mathrm{S} & -0.48185700 & -0.00717200 & 0.00000000 \\ \mathrm{O} & -0.56914500 & 1.42849600 & 0.00000000 \\ \mathrm{O} & -1.67445800 & -0.79614100 & 0.00000000 \\ \mathrm{~N} & 0.82322400 & -0.74437700 & 0.00000000 \\ \mathrm{C} & 2.09848500 & -0.02223300 & 0.00000000 \\ \mathrm{H} & 2.65790500 & -0.33057600 & 0.88204900 \\ \mathrm{H} & 2.65790700 & -0.33058000 & -0.88204600 \\ \mathrm{H} & 1.98925300 & 1.06109700 & -0.00000200\end{array}$

\section{CBS-QB3}

Temperature $=298.150000$

$\mathrm{E}(\mathrm{ZPE})=0.050837$

$\mathrm{E}(\mathrm{SCF})=-641.324930$

$\mathrm{DE}(\mathrm{CBS})=-0.106329$

$\mathrm{DE}(\mathrm{CCSD})=-0.029964$

$\mathrm{DE}($ Empirical $)=-0.047639$

CBS-QB3 $(0 \mathrm{~K})=-642.477991$

CBS-QB3 Enthalpy $=-642.471508$

$$
\begin{aligned}
& \text { Pressure }=1.000000 \\
& E(\text { Thermal })=0.056376 \\
& D E(M P 2)=-1.038802 \\
& D E(M P 34)=-0.015403 \\
& D E(\text { Int })=0.034239
\end{aligned}
$$

CBS-QB3 Energy $=-642.472452$

CBS-QB3 Free Energy $=-642.507270$

$\begin{array}{lrrc}\mathrm{S} & -0.48216200 & -0.00602300 & 0.00000000 \\ \mathrm{O} & -0.55317600 & 1.43795800 & 0.00000000 \\ \mathrm{O} & -1.68542400 & -0.79157900 & 0.00000000 \\ \mathrm{~N} & 0.82168100 & -0.75965500 & 0.00000000 \\ \mathrm{C} & 2.09579000 & -0.02565400 & 0.00000000 \\ \mathrm{H} & 2.65850100 & -0.33143400 & 0.88437300 \\ \mathrm{H} & 2.65850200 & -0.33143500 & -0.88437200 \\ \mathrm{H} & 1.97989100 & 1.05970700 & 0.00000000\end{array}$

\section{M06-2X}

Zero-point correction $=0.052871$ (Hartree/Particle)

Thermal correction to Energy $=0.058179$

Thermal correction to Enthalpy $=0.059124$

Thermal correction to Gibbs Free Energy $=0.024024$

Sum of electronic and zero-point Energies $=-643.170708$

Sum of electronic and thermal Energies $=-643.165400$

Sum of electronic and thermal Enthalpies $=-643.164456$

Sum of electronic and thermal Free Energies $=-643.199556$

$\begin{array}{lrrr}\mathrm{S} & -0.47804400 & -0.00935400 & -0.00000100 \\ \mathrm{O} & -0.54122400 & 1.41578200 & -0.00000500 \\ \mathrm{O} & -1.67590100 & -0.76967300 & 0.00001600 \\ \mathrm{~N} & 0.80945600 & -0.75077200 & -0.00004000 \\ \mathrm{C} & 2.08049200 & -0.02625400 & 0.00001200 \\ \mathrm{H} & 2.63858300 & -0.32986400 & 0.88238000 \\ \mathrm{H} & 2.63947200 & -0.33144000 & -0.88123800 \\ \mathrm{H} & 1.95849200 & 1.05503200 & -0.00100500\end{array}$

\section{MPW1PW91}

Zero-point correction $=0.052287$ (Hartree/Particle)

Thermal correction to Energy $=0.057735$ 
Thermal correction to Enthalpy $=0.058679$

Thermal correction to Gibbs Free Energy $=0.023100$

Sum of electronic and zero-point Energies $=-643.245550$

Sum of electronic and thermal Energies $=-643.240101$

Sum of electronic and thermal Enthalpies $=-643.239157$

Sum of electronic and thermal Free Energies $=-643.274736$

$\begin{array}{lrrr}\mathrm{S} & -0.47837500 & -0.00736000 & 0.00000000 \\ \mathrm{O} & -0.55657600 & 1.42006300 & -0.00000100 \\ \mathrm{O} & -1.66829800 & -0.78518700 & 0.00000000 \\ \mathrm{~N} & 0.81664000 & -0.74590800 & 0.00000000 \\ \mathrm{C} & 2.08062900 & -0.02377400 & 0.00000000 \\ \mathrm{H} & 2.64307100 & -0.32819100 & 0.88112400 \\ \mathrm{H} & 2.64307300 & -0.32819400 & -0.88112200 \\ \mathrm{H} & 1.96659800 & 1.05912600 & -0.00000200\end{array}$

\section{$\mathrm{CH}_{2} \mathrm{NSO}_{2} \mathrm{H}$}

\section{B3LYP/6-311++G(3df,3pd)}

Zero-point correction $=0.050775$ (Hartree/Particle)

Thermal correction to Energy $=0.055875$

Thermal correction to Enthalpy $=0.056819$

Thermal correction to Gibbs Free Energy $=0.021998$

Sum of electronic and zero-point Energies $=-643.312212$

Sum of electronic and thermal Energies $=-643.307112$

Sum of electronic and thermal Enthalpies $=-643.306168$

Sum of electronic and thermal Free Energies $=-643.340989$

$\begin{array}{lrrr}\mathrm{C} & -1.96632700 & 0.00186100 & -0.24289700 \\ \mathrm{H} & -1.81260900 & 0.96742100 & -0.72587200 \\ \mathrm{H} & -2.96800800 & -0.41918600 & -0.23143000 \\ \mathrm{~N} & -1.03713600 & -0.65659200 & 0.31096700 \\ \mathrm{~S} & 0.52035900 & 0.01322200 & 0.22450300 \\ \mathrm{O} & 1.34313900 & -0.91770500 & -0.48453300 \\ \mathrm{O} & 0.49614300 & 1.40620500 & -0.12796600 \\ \mathrm{H} & 0.79853700 & -0.08281300 & 1.54585900\end{array}$

\section{CBS-QB3}

Temperature $=298.150000$

$\mathrm{E}(\mathrm{ZPE})=0.049923$

$\mathrm{E}(\mathrm{SCF})=-641.342541$

$\mathrm{DE}(\mathrm{CBS})=-0.105696$

$\mathrm{DE}(\mathrm{CCSD})=-0.028552$

$\mathrm{DE}($ Empirical $)=-0.047928$

CBS-QB3 $(0 \mathrm{~K})=-642.483946$

CBS-QB3 Enthalpy= -642.477852

$$
\begin{aligned}
& \text { Pressure }=1.000000 \\
& E(\text { Thermal })=0.055073 \\
& D E(M P 2)=-1.019325 \\
& \text { DE }(\text { MP34 })=-0.023649 \\
& \text { DE }(\text { Int })=0.033821
\end{aligned}
$$

CBS-QB3 Energy $=-642.478796$

CBS-QB3 Free Energy= -642.512732

$\begin{array}{lrrr}\mathrm{C} & -1.96788400 & 0.00083600 & -0.24829400 \\ \mathrm{H} & -1.80229600 & 0.96460100 & -0.73669300 \\ \mathrm{H} & -2.97567800 & -0.41233600 & -0.23955700 \\ \mathrm{~N} & -1.04760100 & -0.66594500 & 0.31841500\end{array}$




$\begin{array}{lrrr}\mathrm{S} & 0.52467800 & 0.01511000 & 0.22365100 \\ \mathrm{O} & 1.35146700 & -0.92167900 & -0.48605900 \\ \mathrm{O} & 0.48959400 & 1.41476700 & -0.12489400 \\ \mathrm{H} & 0.79514600 & -0.08214300 & 1.54632100\end{array}$

\section{M06-2X}

Zero-point correction $=0.052100$ (Hartree/Particle)

Thermal correction to Energy $=0.057035$

Thermal correction to Enthalpy $=0.057980$

Thermal correction to Gibbs Free Energy $=0.023570$

Sum of electronic and zero-point Energies $=-643.174868$

Sum of electronic and thermal Energies $=-643.169933$

Sum of electronic and thermal Enthalpies $=-643.168989$

Sum of electronic and thermal Free Energies $=-643.203398$

$\begin{array}{lrrr}\mathrm{C} & -1.94866400 & -0.01035000 & -0.23327700 \\ \mathrm{H} & -1.79450900 & 0.97851800 & -0.66537600 \\ \mathrm{H} & -2.94586300 & -0.43976800 & -0.24084300 \\ \mathrm{~N} & -1.01444900 & -0.68564900 & 0.28481700 \\ \mathrm{~S} & 0.51468800 & 0.01571300 & 0.22523400 \\ \mathrm{O} & 1.36548800 & -0.88110300 & -0.47779700 \\ \mathrm{O} & 0.44876400 & 1.39903000 & -0.12650500 \\ \mathrm{H} & 0.78447400 & -0.07193600 & 1.54283200\end{array}$

\section{MPW1PW91}

Zero-point correction $=0.051479$ (Hartree/Particle)

Thermal correction to Energy $=0.056507$

Thermal correction to Enthalpy= 0.057451

Thermal correction to Gibbs Free Energy $=0.022808$

Sum of electronic and zero-point Energies $=-643.247915$

Sum of electronic and thermal Energies $=-643.242887$

Sum of electronic and thermal Enthalpies $=-643.241943$

Sum of electronic and thermal Free Energies $=-643.276586$

$\begin{array}{lrcc}\mathrm{C} & -1.94916400 & -0.00565400 & -0.23762100 \\ \mathrm{H} & -1.79604600 & 0.97404100 & -0.69205100 \\ \mathrm{H} & -2.94814700 & -0.43287400 & -0.23840000 \\ \mathrm{~N} & -1.01809200 & -0.67176400 & 0.29723100 \\ \mathrm{~S} & 0.51378000 & 0.01478300 & 0.22366100 \\ \mathrm{O} & 1.35149900 & -0.89611800 & -0.47976900 \\ \mathrm{O} & 0.46833800 & 1.40054100 & -0.12613200 \\ \mathrm{H} & 0.78664400 & -0.07680700 & 1.54419600\end{array}$

anti-CH $\mathrm{CH}_{3} \mathrm{~S}(\mathrm{O}) \mathrm{NO}$

B3LYP/6-311++G(3df,3pd)

Zero-point correction $=0.047479$ (Hartree/Particle)

Thermal correction to Energy $=0.054093$

Thermal correction to Enthalpy $=0.055037$

Thermal correction to Gibbs Free Energy $=0.016767$

Sum of electronic and zero-point Energies $=-643.264683$ 
Sum of electronic and thermal Energies $=-643.258070$

Sum of electronic and thermal Enthalpies $=-643.257126$

Sum of electronic and thermal Free Energies $=-643.295396$

$\begin{array}{lrcc}\mathrm{C} & -0.59227100 & 1.51024700 & 0.18557300 \\ \mathrm{H} & 0.19681300 & 2.08504300 & -0.29715500 \\ \mathrm{H} & -1.56638400 & 1.91760100 & -0.07633900 \\ \mathrm{H} & -0.47211000 & 1.49646400 & 1.26783800 \\ \mathrm{~S} & -0.54591700 & -0.19748900 & -0.44133700 \\ \mathrm{O} & -1.50438900 & -0.98554400 & 0.35889000 \\ \mathrm{~N} & 1.26557700 & -0.42768800 & 0.48697300 \\ \mathrm{O} & 2.16325700 & -0.06532600 & -0.15329000\end{array}$

\section{CBS-QB3}

Temperature $=298.150000$

$\mathrm{E}(\mathrm{ZPE})=0.046727$

$\mathrm{E}(\mathrm{SCF})=-641.256390$

$\mathrm{DE}(\mathrm{CBS})=-0.105778$

$\mathrm{DE}(\mathrm{CCSD})=-0.032587$

$\mathrm{DE}($ Empirical $)=-0.046357$

CBS-QB3 $(0 \mathrm{~K})=-642.427335$

CBS-QB3 Enthalpy $=-642.419634$

$$
\begin{aligned}
& \text { Pressure }=1.000000 \\
& E(\text { Thermal })=0.053484 \\
& D E(M P 2)=-1.038510 \\
& D E(M P 34)=-0.028867 \\
& D E(\text { Int })=0.034426
\end{aligned}
$$

CBS-QB3 Energy $=-642.420578$

CBS-QB3 Free Energy= -642.458354

$\begin{array}{lccc}\mathrm{C} & -0.56905000 & 1.51896200 & 0.18005500 \\ \mathrm{H} & 0.25301800 & 2.07074900 & -0.28001200 \\ \mathrm{H} & -1.52531900 & 1.95665400 & -0.10836000 \\ \mathrm{H} & -0.48105600 & 1.49970300 & 1.26797400 \\ \mathrm{~S} & -0.55574700 & -0.20450000 & -0.43770400 \\ \mathrm{O} & -1.56015200 & -0.95644000 & 0.35615300 \\ \mathrm{~N} & 1.30242500 & -0.44194000 & 0.50502300 \\ \mathrm{O} & 2.17798200 & -0.07797100 & -0.16763300\end{array}$

\section{M06-2X}

Zero-point correction $=0.048627$ (Hartree/Particle)

Thermal correction to Energy $=0.054870$

Thermal correction to Enthalpy $=0.055814$

Thermal correction to Gibbs Free Energy $=0.018694$

Sum of electronic and zero-point Energies $=-643.120500$

Sum of electronic and thermal Energies $=-643.114256$

Sum of electronic and thermal Enthalpies $=-643.113312$

Sum of electronic and thermal Free Energies $=-643.150432$

$\begin{array}{lccc}\mathrm{C} & -0.43926500 & 1.51021800 & 0.18879300 \\ \mathrm{H} & 0.42883000 & 2.00471100 & -0.24115800 \\ \mathrm{H} & -1.35474600 & 2.00213500 & -0.12805000 \\ \mathrm{H} & -0.38768500 & 1.46928000 & 1.27513300 \\ \mathrm{~S} & -0.51484600 & -0.17948100 & -0.43465300 \\ \mathrm{O} & -1.53403600 & -0.88225700 & 0.34747300 \\ \mathrm{~N} & 1.14682400 & -0.56075900 & 0.43804800 \\ \mathrm{O} & 2.05390700 & -0.08529600 & -0.11629400\end{array}$




\section{MPW1PW91}

Zero-point correction $=0.048145$ (Hartree/Particle)

Thermal correction to Energy $=0.054607$

Thermal correction to Enthalpy $=0.055552$

Thermal correction to Gibbs Free Energy $=0.017758$

Sum of electronic and zero-point Energies $=-643.194473$

Sum of electronic and thermal Energies $=-643.188010$

Sum of electronic and thermal Enthalpies $=-643.187066$

Sum of electronic and thermal Free Energies $=-643.224859$

$\begin{array}{lccc}\mathrm{C} & -0.51746400 & 1.50440200 & 0.18541100 \\ \mathrm{H} & 0.30723500 & 2.04378200 & -0.27734800 \\ \mathrm{H} & -1.46575200 & 1.95281600 & -0.10172100 \\ \mathrm{H} & -0.42655200 & 1.48633600 & 1.27036200 \\ \mathrm{~S} & -0.52919200 & -0.18689300 & -0.43460100 \\ \mathrm{O} & -1.51633000 & -0.93819600 & 0.34811900 \\ \mathrm{~N} & 1.20391600 & -0.47973400 & 0.46888600 \\ \mathrm{O} & 2.10751900 & -0.08191900 & -0.13966300\end{array}$

\section{syn- $\mathrm{CH}_{3} \mathrm{~S}(\mathrm{O}) \mathrm{NO}$}

\section{B3LYP/6-311++G(3df,3pd)}

Zero-point correction $=0.047739$ (Hartree/Particle $)$

Thermal correction to Energy $=0.054212$

Thermal correction to Enthalpy $=0.055156$

Thermal correction to Gibbs Free Energy $=0.017558$

Sum of electronic and zero-point Energies $=-643.269847$

Sum of electronic and thermal Energies $=-643.263373$

Sum of electronic and thermal Enthalpies $=-643.262429$

Sum of electronic and thermal Free Energies $=-643.300027$

$\begin{array}{lccc}\mathrm{C} & -1.37544800 & -1.04944100 & 0.39601000 \\ \mathrm{H} & -1.31143500 & -1.95248000 & -0.21010200 \\ \mathrm{H} & -0.89434400 & -1.19116400 & 1.36202900 \\ \mathrm{H} & -2.41602600 & -0.76511100 & 0.53419300 \\ \mathrm{~S} & -0.54850000 & 0.30200000 & -0.49982900 \\ \mathrm{O} & -0.31598300 & 1.41473100 & 0.44882500 \\ \mathrm{~N} & 1.24290300 & -0.74181100 & -0.32685500 \\ \mathrm{O} & 1.93475600 & -0.09397200 & 0.32905800\end{array}$

\section{CBS-QB3}

Temperature $=298.150000$

Pressure $=1.000000$

$\mathrm{E}(\mathrm{ZPE})=0.047091$

$\mathrm{E}(\mathrm{SCF})=-641.257611$

$\mathrm{E}($ Thermal $)=0.053635$

$\mathrm{DE}(\mathrm{CBS})=-0.106199$

$\mathrm{DE}(\mathrm{MP} 2)=-1.046477$

$\mathrm{DE}(\mathrm{MP} 34)=-0.026460$

$\mathrm{DE}(\mathrm{CCSD})=-0.031900$

$\mathrm{DE}($ Empirical $)=-0.046223$

CBS-QB3 $(0 \mathrm{~K})=-642.433220$

$\mathrm{DE}(\mathrm{Int})=0.034559$

CBS-QB3 Enthalpy= -642.425731

CBS-QB3 Energy $=-642.426675$

CBS-QB3 Free Energy= -642.463510 


$\begin{array}{lccc}\mathrm{C} & -1.39634300 & -1.03485800 & 0.40884000 \\ \mathrm{H} & -1.35379800 & -1.94266900 & -0.19679700 \\ \mathrm{H} & -0.88315200 & -1.17266600 & 1.36158900 \\ \mathrm{H} & -2.43285200 & -0.74437800 & 0.58150100 \\ \mathrm{~S} & -0.56978800 & 0.31886700 & -0.50884400 \\ \mathrm{O} & -0.24824900 & 1.40122300 & 0.46346000 \\ \mathrm{~N} & 1.26193900 & -0.76513000 & -0.35209300 \\ \mathrm{O} & 1.91461000 & -0.11086200 & 0.33739200\end{array}$

\section{M06-2X}

Zero-point correction $=0.048821$ (Hartree/Particle)

Thermal correction to Energy $=0.054981$

Thermal correction to Enthalpy $=0.055925$

Thermal correction to Gibbs Free Energy $=0.019155$

Sum of electronic and zero-point Energies $=-643.125505$

Sum of electronic and thermal Energies $=-643.119345$

Sum of electronic and thermal Enthalpies $=-643.118401$

Sum of electronic and thermal Free Energies $=-643.155172$

$\begin{array}{lrrr}\mathrm{C} & -1.34605500 & -1.02588800 & 0.38933400 \\ \mathrm{H} & -1.26567600 & -1.94284100 & -0.19122400 \\ \mathrm{H} & -0.88378200 & -1.13526100 & 1.36845700 \\ \mathrm{H} & -2.38694500 & -0.73315500 & 0.49240200 \\ \mathrm{~S} & -0.50029600 & 0.29564900 & -0.49505300 \\ \mathrm{O} & -0.30871400 & 1.41045700 & 0.43898400 \\ \mathrm{~N} & 1.14965000 & -0.72792000 & -0.32281300 \\ \mathrm{O} & 1.87995500 & -0.11900200 & 0.33287800\end{array}$

\section{MPW1PW91}

Zero-point correction $=0.048407$ (Hartree/Particle)

Thermal correction to Energy $=0.054741$

Thermal correction to Enthalpy $=0.055685$

Thermal correction to Gibbs Free Energy $=0.018465$

Sum of electronic and zero-point Energies $=-643.199763$

Sum of electronic and thermal Energies $=-643.193429$

Sum of electronic and thermal Enthalpies $=-643.192485$

Sum of electronic and thermal Free Energies $=-643.229705$

$\begin{array}{lrrr}\mathrm{C} & -1.36032300 & -1.03095300 & 0.38910100 \\ \mathrm{H} & -1.27716500 & -1.94326200 & -0.19990000 \\ \mathrm{H} & -0.90673200 & -1.15529800 & 1.37060300 \\ \mathrm{H} & -2.40464300 & -0.74689500 & 0.49161700 \\ \mathrm{~S} & -0.51939500 & 0.29631200 & -0.49255400 \\ \mathrm{O} & -0.30917600 & 1.41584200 & 0.43700200 \\ \mathrm{~N} & 1.18493500 & -0.73882500 & -0.31627500 \\ \mathrm{O} & 1.90495800 & -0.10809800 & 0.32523100\end{array}$

\section{$\mathrm{CH}_{3} \mathrm{SO}_{2} \mathrm{~N}($ Singlet)}

B3LYP/6-311++G(3df,3pd)

Zero-point correction $=0.049640$ (Hartree/Particle) 
Thermal correction to Energy $=0.055058$

Thermal correction to Enthalpy= 0.056002

Thermal correction to Gibbs Free Energy $=0.021078$

Sum of electronic and zero-point Energies $=-643.209791$

Sum of electronic and thermal Energies $=-643.204374$

Sum of electronic and thermal Enthalpies $=-643.203429$

Sum of electronic and thermal Free Energies $=-643.238353$

$\begin{array}{lccc}\mathrm{N} & -1.20122400 & -0.38886400 & -0.89438400 \\ \mathrm{~S} & -0.01661300 & 0.16088600 & -0.05651300 \\ \mathrm{O} & 0.38349500 & 1.52638900 & 0.06464400 \\ \mathrm{O} & -1.03066300 & -0.48633100 & 0.87371100 \\ \mathrm{C} & 1.44583600 & -0.84770900 & -0.03812400 \\ \mathrm{H} & 2.00528700 & -0.61320300 & 0.86493000 \\ \mathrm{H} & 2.03594000 & -0.58651100 & -0.91382100 \\ \mathrm{H} & 1.13547800 & -1.88662800 & -0.06432400\end{array}$

\section{CBS-QB3}

Temperature $=298.150000$

Pressure $=1.000000$

$\mathrm{E}(\mathrm{ZPE})=0.048676$

$\mathrm{E}($ Thermal $)=0.054240$

$\mathrm{E}(\mathrm{SCF})=-641.222479$

$\mathrm{DE}(\mathrm{MP} 2)=-1.031806$

$\mathrm{DE}(\mathrm{CBS})=-0.106748$

$\mathrm{DE}(\mathrm{MP} 34)=-0.027331$

$\mathrm{DE}(\mathrm{CCSD})=-0.032127$

$\mathrm{DE}($ Empirical $)=-0.047336$

CBS-QB3 $(0 \mathrm{~K})=-642.384835$

$\mathrm{DE}(\operatorname{Int})=0.034316$

CBS-QB3 Enthalpy= -642.378327

CBS-QB3 Energy= -642.379271

CBS-QB3 Free Energy= -642.413565

$\begin{array}{lccc}\mathrm{N} & -1.20206900 & -0.37724500 & -0.91352700 \\ \mathrm{~S} & -0.02087700 & 0.16375200 & -0.05480100 \\ \mathrm{O} & 0.38866400 & 1.53244000 & 0.07359300 \\ \mathrm{O} & -1.02658600 & -0.50125100 & 0.88775500 \\ \mathrm{C} & 1.44631200 & -0.85343000 & -0.04613300 \\ \mathrm{H} & 2.01163700 & -0.62259000 & 0.85723200 \\ \mathrm{H} & 2.03306700 & -0.59265600 & -0.92750500 \\ \mathrm{H} & 1.12930900 & -1.89300200 & -0.07220900\end{array}$

M06-2X

Zero-point correction $=0.050659$ (Hartree/Particle)

Thermal correction to Energy $=0.055906$

Thermal correction to Enthalpy $=0.056850$

Thermal correction to Gibbs Free Energy $=0.022242$

Sum of electronic and zero-point Energies $=-643.071557$

Sum of electronic and thermal Energies $=-643.066310$

Sum of electronic and thermal Enthalpies $=-643.065366$

Sum of electronic and thermal Free Energies= -643.099974

$\begin{array}{lccc}\mathrm{N} & -1.22177700 & -0.36035000 & -0.84769200 \\ \mathrm{~S} & -0.00179200 & 0.16493300 & -0.06429900 \\ \mathrm{O} & 0.43733600 & 1.50846800 & 0.06525400 \\ \mathrm{O} & -1.05037700 & -0.45217200 & 0.83951700\end{array}$




$\begin{array}{lccc}\mathrm{C} & 1.40909800 & -0.88909600 & -0.03177100 \\ \mathrm{H} & 1.96146400 & -0.67511800 & 0.87968500 \\ \mathrm{H} & 2.01699000 & -0.64498100 & -0.89905700 \\ \mathrm{H} & 1.05239000 & -1.91216300 & -0.06554300\end{array}$

\section{MPW1PW91}

Zero-point correction $=0.050293$ (Hartree/Particle)

Thermal correction to Energy= 0.055623

Thermal correction to Enthalpy $=0.056567$

Thermal correction to Gibbs Free Energy $=0.021820$

Sum of electronic and zero-point Energies $=-643.147244$

Sum of electronic and thermal Energies $=-643.141914$

Sum of electronic and thermal Enthalpies $=-643.140970$

Sum of electronic and thermal Free Energies $=-643.175718$

$\begin{array}{lccc}\mathrm{N} & -1.20741500 & -0.36785800 & -0.86930300 \\ \mathrm{~S} & -0.00757200 & 0.16224800 & -0.05939800 \\ \mathrm{O} & 0.42043800 & 1.51132800 & 0.06363900 \\ \mathrm{O} & -1.04345100 & -0.46086500 & 0.85267500 \\ \mathrm{C} & 1.41445400 & -0.87323200 & -0.03313800 \\ \mathrm{H} & 1.98041100 & -0.64982300 & 0.86849000 \\ \mathrm{H} & 2.01299000 & -0.63183200 & -0.90864400 \\ \mathrm{H} & 1.07703500 & -1.90361600 & -0.05603800\end{array}$

\section{$\mathrm{CH}_{3} \mathrm{SO}_{2} \mathrm{~N}$ (Triplet)}

B3LYP/6-311++G(3df,3pd)

Zero-point correction $=0.048832$ (Hartree/Particle)

Thermal correction to Energy $=0.054420$

Thermal correction to Enthalpy $=0.055365$

Thermal correction to Gibbs Free Energy $=0.019056$

Sum of electronic and zero-point Energies $=-643.232653$

Sum of electronic and thermal Energies $=-643.227065$

Sum of electronic and thermal Enthalpies $=-643.226121$

Sum of electronic and thermal Free Energies= -643.262430

$\begin{array}{lrrc}\mathrm{S} & -0.13973600 & 0.00000000 & -0.07239700 \\ \mathrm{O} & -0.59889700 & 1.25018500 & -0.61494500 \\ \mathrm{O} & -0.59889800 & -1.25018400 & -0.61494600 \\ \mathrm{~N} & -0.55965300 & 0.00000000 & 1.56404300 \\ \mathrm{C} & 1.63648900 & -0.00000100 & 0.00744400 \\ \mathrm{H} & 1.97823500 & 0.00000000 & -1.02705200 \\ \mathrm{H} & 1.96926300 & -0.90068900 & 0.51578100 \\ \mathrm{H} & 1.96926400 & 0.90068700 & 0.51578200\end{array}$

\section{CBS-QB3}

Temperature $=298.150000$

$\mathrm{E}(\mathrm{ZPE})=0.047964$

$\mathrm{E}(\mathrm{SCF})=-641.310806$

$\mathrm{DE}(\mathrm{CBS})=-0.102076$

$\mathrm{DE}(\mathrm{CCSD})=-0.025699$
Pressure $=1.000000$

$\mathrm{E}($ Thermal $)=0.053678$

$\mathrm{DE}(\mathrm{MP} 2)=-0.957001$

$\mathrm{DE}(\mathrm{MP} 34)=-0.036954$

$\mathrm{DE}(\mathrm{Int})=0.031822$ 
$\mathrm{DE}($ Empirical $)=-0.045104$

CBS-QB3 $(0 \mathrm{~K})=-642.397853$

CBS-QB3 Enthalpy= -642.391196

CBS-QB3 Energy $=-642.392140$

CBS-QB3 Free Energy= -642.427782

$\begin{array}{lrrr}\mathrm{S} & -0.14416400 & 0.00000000 & -0.07404600 \\ \mathrm{O} & -0.60343700 & 1.25864300 & -0.61344500 \\ \mathrm{O} & -0.60343700 & -1.25864100 & -0.61344900 \\ \mathrm{~N} & -0.54551600 & -0.00000200 & 1.57660200 \\ \mathrm{C} & 1.64153300 & 0.00000000 & -0.00177700 \\ \mathrm{H} & 1.98022000 & 0.00000100 & -1.03998200 \\ \mathrm{H} & 1.97540700 & -0.90312600 & 0.50716200 \\ \mathrm{H} & 1.97540700 & 0.90312500 & 0.50716400\end{array}$

M06-2X

Zero-point correction $=0.049767$ (Hartree/Particle)

Thermal correction to Energy $=0.055222$

Thermal correction to Enthalpy $=0.056166$

Thermal correction to Gibbs Free Energy $=0.020103$

Sum of electronic and zero-point Energies $=-643.093678$

Sum of electronic and thermal Energies $=-643.088223$

Sum of electronic and thermal Enthalpies $=-643.087278$

Sum of electronic and thermal Free Energies= -643.123342

$\begin{array}{lrrr}\mathrm{S} & 0.14180800 & 0.00000000 & -0.07810400 \\ \mathrm{O} & 0.60571400 & -1.24466400 & -0.60084300 \\ \mathrm{O} & 0.60571500 & 1.24466400 & -0.60084100 \\ \mathrm{~N} & 0.51748300 & -0.00000200 & 1.57563500 \\ \mathrm{C} & -1.62114100 & 0.00000100 & -0.01591600 \\ \mathrm{H} & -1.95174300 & 0.00000100 & -1.05298900 \\ \mathrm{H} & -1.95207400 & 0.90152300 & 0.49109100 \\ \mathrm{H} & -1.95207500 & -0.90152200 & 0.49109000\end{array}$

\section{MPW1PW91}

Zero-point correction $=0.049461$ (Hartree/Particle)

Thermal correction to Energy $=0.054967$

Thermal correction to Enthalpy $=0.055911$

Thermal correction to Gibbs Free Energy $=0.019774$

Sum of electronic and zero-point Energies $=-643.172883$

Sum of electronic and thermal Energies $=-643.167377$

Sum of electronic and thermal Enthalpies $=-643.166433$

Sum of electronic and thermal Free Energies $=-643.202571$

$\begin{array}{lrrr}\mathrm{S} & 0.13950600 & 0.00000000 & -0.07341000 \\ \mathrm{O} & 0.59967300 & -1.24424700 & -0.60654600 \\ \mathrm{O} & 0.59967600 & 1.24424800 & -0.60654300 \\ \mathrm{~N} & 0.53854800 & -0.00000300 & 1.56171200 \\ \mathrm{C} & -1.62091900 & 0.00000200 & -0.00304200 \\ \mathrm{H} & -1.95648500 & 0.00000100 & -1.03925900 \\ \mathrm{H} & -1.95736200 & 0.90076000 & 0.50239900 \\ \mathrm{H} & -1.95736300 & -0.90075600 & 0.50240000\end{array}$




\section{$\mathrm{HSO}_{2} \mathrm{~N}($ Singlet $)$}

\section{B3LYP/6-311++(3df,3pd)}

Zero-point correction $=0.021150$ (Hartree/Particle)

Thermal correction to Energy $=0.025024$

Thermal correction to Enthalpy $=0.025968$

Thermal correction to Gibbs Free Energy $=-0.005339$

Sum of electronic and zero-point Energies $=-603.892049$

Sum of electronic and thermal Energies $=-603.888175$

Sum of electronic and thermal Enthalpies $=-603.887231$

Sum of electronic and thermal Free Energies $=-603.918538$

$\begin{array}{lccc}\mathrm{S} & -0.17363400 & 0.03727000 & 0.23173700 \\ \mathrm{H} & -0.30711500 & -0.14398200 & 1.56497000 \\ \mathrm{O} & 0.97702700 & -0.85752900 & -0.17946500 \\ \mathrm{O} & -1.48277500 & -0.01168800 & -0.32161100 \\ \mathrm{~N} & 1.01875000 & 0.92877200 & -0.18059300\end{array}$

\section{CBS-QB3}

Temperature $=298.150000$

Pressure $=1.000000$

$\mathrm{E}(\mathrm{ZPE})=0.020594$

$\mathrm{E}(\mathrm{SCF})=-602.155380$

$\mathrm{DE}(\mathrm{CBS})=-0.090244$

$\mathrm{DE}(\mathrm{CCSD})=-0.027627$

$\mathrm{DE}($ Empirical $)=-0.038445$

CBS-QB3 $(0 \mathrm{~K})=-603.140054$

CBS-QB3 Enthalpy $=-603.135155$

$\mathrm{E}($ Thermal $)=0.024548$

$\mathrm{DE}(\mathrm{MP} 2)=-0.861360$

$\mathrm{DE}(\mathrm{MP} 34)=-0.016200$

$\mathrm{DE}(\mathrm{Int})=0.028606$

$\begin{array}{lrcc}\mathrm{S} & -0.16949000 & 0.03328900 & 0.22919100 \\ \mathrm{H} & -0.29955400 & -0.16309900 & 1.55977800 \\ \mathrm{O} & 0.98354400 & -0.87132200 & -0.18329000 \\ \mathrm{O} & -1.48924400 & -0.01213700 & -0.31643500 \\ \mathrm{~N} & 1.00814100 & 0.95687800 & -0.17557600\end{array}$

\section{M06-2X}

Zero-point correction $=0.022071$ (Hartree/Particle)

Thermal correction to Energy $=0.025811$

Thermal correction to Enthalpy $=0.026755$

Thermal correction to Gibbs Free Energy $=-0.004294$

Sum of electronic and zero-point Energies $=-603.773643$

Sum of electronic and thermal Energies $=-603.769903$

Sum of electronic and thermal Enthalpies $=-603.768959$

Sum of electronic and thermal Free Energies $=-603.800008$

$\begin{array}{lccc}\mathrm{S} & -0.18444200 & 0.04494900 & 0.23495900 \\ \mathrm{H} & -0.31873600 & -0.13782400 & 1.56156000 \\ \mathrm{O} & 0.98146200 & -0.82388400 & -0.17330600 \\ \mathrm{O} & -1.47856800 & -0.01671400 & -0.32942800 \\ \mathrm{~N} & 1.03523600 & 0.87763300 & -0.18557700\end{array}$




\section{MPW1PW91}

Zero-point correction $=0.021651$ (Hartree/Particle)

Thermal correction to Energy $=0.025465$

Thermal correction to Enthalpy $=0.026409$

Thermal correction to Gibbs Free Energy $=-0.004773$

Sum of electronic and zero-point Energies $=-603.838850$

Sum of electronic and thermal Energies $=-603.835035$

Sum of electronic and thermal Enthalpies $=-603.834091$

Sum of electronic and thermal Free Energies $=-603.865273$

$\begin{array}{lccc}\mathrm{S} & -0.17883200 & 0.03996000 & 0.23210000 \\ \mathrm{H} & -0.31197600 & -0.14785700 & 1.56150600 \\ \mathrm{O} & 0.98008600 & -0.83601100 & -0.17723600 \\ \mathrm{O} & -1.47876900 & -0.01411200 & -0.32412900 \\ \mathrm{~N} & 1.02325000 & 0.90135400 & -0.18059800\end{array}$

\section{$\mathrm{HSO}_{2} \mathrm{~N}$ (Triplet)}

B3LYP/6-311++G(3df,3pd)

Zero-point correction $=0.020464$ (Hartree/Particle)

Thermal correction to Energy $=0.024487$

Thermal correction to Enthalpy $=0.025431$

Thermal correction to Gibbs Free Energy $=-0.007352$

Sum of electronic and zero-point Energies $=-603.913600$

Sum of electronic and thermal Energies $=-603.909578$

Sum of electronic and thermal Enthalpies $=-603.908633$

Sum of electronic and thermal Free Energies $=-603.941416$

$\begin{array}{lrcc}\mathrm{S} & 0.00000000 & -0.07076800 & 0.20022700 \\ \mathrm{H} & -0.00000100 & 0.04065400 & 1.55713500 \\ \mathrm{O} & 1.25479000 & -0.62068300 & -0.21807500 \\ \mathrm{O} & -1.25479600 & -0.62067100 & -0.21807500 \\ \mathrm{~N} & 0.00000800 & 1.57464000 & -0.18165200\end{array}$

\section{CBS-QB3}

Temperature $=298.150000$

$\mathrm{E}(\mathrm{ZPE})=0.019962$

$\mathrm{E}(\mathrm{SCF})=-602.244023$

$\mathrm{DE}(\mathrm{CBS})=-0.085285$

$\mathrm{DE}(\mathrm{CCSD})=-0.020788$

$\mathrm{DE}($ Empirical $)=-0.035746$

CBS-QB3 $(0 \mathrm{~K})=-603.150788$

CBS-QB3 Enthalpy= -603.145764

$$
\begin{aligned}
& \text { Pressure }=1.000000 \\
& E(\text { Thermal })=0.024042 \\
& D E(M P 2)=-0.785635 \\
& D E(M P 34)=-0.025204 \\
& D E(\text { Int })=0.025932
\end{aligned}
$$

CBS-QB3 Energy= -603.146708

CBS-QB3 Free Energy= -603.178657

$\begin{array}{lrcc}\mathrm{S} & 0.00000000 & -0.07233200 & 0.19694100 \\ \mathrm{H} & 0.00000000 & 0.04890700 & 1.55287600 \\ \mathrm{O} & 1.26319300 & -0.62374100 & -0.21523800 \\ \mathrm{O} & -1.26318700 & -0.62375200 & -0.21523800 \\ \mathrm{~N} & -0.00000800 & 1.58404900 & -0.18001700\end{array}$

M06-2X

Zero-point correction $=0.021585$ (Hartree/Particle) 
Thermal correction to Energy $=0.025485$

Thermal correction to Enthalpy= 0.026430

Thermal correction to Gibbs Free Energy $=-0.006134$

Sum of electronic and zero-point Energies $=-603.794401$

Sum of electronic and thermal Energies $=-603.790500$

Sum of electronic and thermal Enthalpies $=-603.789556$

Sum of electronic and thermal Free Energies $=-603.822120$

$\begin{array}{lrcc}\mathrm{S} & 0.00000600 & -0.07716800 & 0.20001500 \\ \mathrm{H} & 0.00000000 & 0.03245600 & 1.55014900 \\ \mathrm{O} & 1.24870000 & -0.61304000 & -0.21925900 \\ \mathrm{O} & -1.24862700 & -0.61318300 & -0.21925800 \\ \mathrm{~N} & -0.00009700 & 1.57314500 & -0.17746400\end{array}$

\section{MPW1PW91}

Zero-point correction $=0.020989$ (Hartree/Particle)

Thermal correction to Energy $=0.024953$

Thermal correction to Enthalpy $=0.025897$

Thermal correction to Gibbs Free Energy $=-0.006773$

Sum of electronic and zero-point Energies $=-603.863261$

Sum of electronic and thermal Energies $=-603.859298$

Sum of electronic and thermal Enthalpies $=-603.858353$

Sum of electronic and thermal Free Energies $=-603.891023$

$\begin{array}{lrrc}\mathrm{S} & 0.00000000 & -0.07205800 & 0.19867500 \\ \mathrm{H} & 0.00000000 & 0.04328100 & 1.55253200 \\ \mathrm{O} & 1.24908300 & -0.61625800 & -0.21687800 \\ \mathrm{O} & -1.24908100 & -0.61626300 & -0.21687800 \\ \mathrm{~N} & -0.00000400 & 1.56711700 & -0.18018400\end{array}$

\section{$\mathrm{CF}_{3} \mathrm{SO}_{2} \mathrm{~N}$ (Singlet)}

\section{B3LYP/6-311++G(3df,3pd)}

Zero-point correction $=0.026409$ (Hartree/Particle)

Thermal correction to Energy= 0.033893

Thermal correction to Enthalpy $=0.034837$

Thermal correction to Gibbs Free Energy $=-0.006547$

Sum of electronic and zero-point Energies $=-941.040611$

Sum of electronic and thermal Energies $=-941.033127$

Sum of electronic and thermal Enthalpies $=-941.032182$

Sum of electronic and thermal Free Energies $=-941.073567$

$\begin{array}{lrrr}\mathrm{S} & -0.85153800 & 0.20816600 & -0.05694900 \\ \mathrm{O} & -1.03508800 & 1.61206100 & 0.06168700 \\ \mathrm{O} & -1.42491800 & -0.82938600 & 0.87917300 \\ \mathrm{~N} & -1.63582000 & -0.81319400 & -0.90887500 \\ \mathrm{C} & 1.00524700 & -0.07433500 & -0.01266000 \\ \mathrm{~F} & 1.49202700 & 0.42194400 & 1.11633200 \\ \mathrm{~F} & 1.54357800 & 0.55813300 & -1.04844600 \\ \mathrm{~F} & 1.26705100 & -1.36382100 & -0.08762200\end{array}$




\section{CBS-QB3}

Temperature $=298.150000$

$\mathrm{E}(\mathrm{ZPE})=0.025836$

$\mathrm{E}(\mathrm{SCF})=-937.866894$

$\mathrm{DE}(\mathrm{CBS})=-0.188747$

$\mathrm{DE}(\mathrm{CCSD})=-0.044187$

$\mathrm{DE}($ Empirical $)=-0.082999$

CBS-QB3 $(0 \mathrm{~K})=-939.877065$

CBS-QB3 Enthalpy= -939.868516

$\begin{array}{lr}\mathrm{S} & -0.85154500 \\ \mathrm{O} & -1.03921300 \\ \mathrm{O} & -1.41600600 \\ \mathrm{~N} & -1.62694900 \\ \mathrm{C} & 1.00245700 \\ \mathrm{~F} & 1.49452700 \\ \mathrm{~F} & 1.53698000 \\ \mathrm{~F} & 1.26186700\end{array}$

Pressure $=1.000000$

$\mathrm{E}($ Thermal $)=0.033441$

$\mathrm{DE}(\mathrm{MP} 2)=-1.755414$

$\mathrm{DE}(\mathrm{MP} 34)=-0.018299$

$\mathrm{DE}(\operatorname{Int})=0.053639$

CBS-QB3 Energy= -939.869460

CBS-QB3 Free Energy= -939.910242

$\begin{array}{rr}0.20687200 & -0.05331900 \\ 1.61696500 & 0.06637100 \\ -0.84054300 & 0.89293500 \\ -0.80922300 & -0.93056400 \\ -0.07256400 & -0.01299500 \\ 0.43260000 & 1.11385000 \\ 0.55403500 & -1.05894400 \\ -1.36678800 & -0.08040000\end{array}$

M06-2X

Zero-point correction $=0.027860$ (Hartree/Particle)

Thermal correction to Energy $=0.035094$

Thermal correction to Enthalpy $=0.036038$

Thermal correction to Gibbs Free Energy= -0.004860

Sum of electronic and zero-point Energies $=-940.824107$

Sum of electronic and thermal Energies $=-940.816873$

Sum of electronic and thermal Enthalpies $=-940.815929$

Sum of electronic and thermal Free Energies $=-940.856827$

$\begin{array}{lrrr}\mathrm{S} & -0.83275900 & 0.22568400 & -0.06818700 \\ \mathrm{O} & -1.00423200 & 1.62064300 & 0.06419000 \\ \mathrm{O} & -1.41049900 & -0.82608600 & 0.84230300 \\ \mathrm{~N} & -1.63427200 & -0.81649300 & -0.85726200 \\ \mathrm{C} & 0.99118300 & -0.07959100 & -0.01341700 \\ \mathrm{~F} & 1.46475500 & 0.37480600 & 1.12788100 \\ \mathrm{~F} & 1.54761500 & 0.56863000 & -1.01665500 \\ \mathrm{~F} & 1.22482900 & -1.36281400 & -0.12007300\end{array}$

\section{MPW1PW91}

Zero-point correction $=0.027257$ (Hartree/Particle)

Thermal correction to Energy $=0.034613$

Thermal correction to Enthalpy $=0.035557$

Thermal correction to Gibbs Free Energy $=-0.005567$

Sum of electronic and zero-point Energies $=-940.910264$

Sum of electronic and thermal Energies $=-940.902907$

Sum of electronic and thermal Enthalpies $=-940.901963$

Sum of electronic and thermal Free Energies $=-940.943087$

$\begin{array}{llrr}\mathrm{S} & -0.83877200 & 0.21624700 & -0.06142900 \\ \mathrm{O} & -1.01626200 & 1.61320900 & 0.06315800\end{array}$




$\begin{array}{crrr}\mathrm{O} & -1.41869300 & -0.82664700 & 0.85641100 \\ \mathrm{~N} & -1.63112700 & -0.81092600 & -0.88067300 \\ \mathrm{C} & 0.99592300 & -0.07739400 & -0.01258400 \\ \mathrm{~F} & 1.47763200 & 0.40479700 & 1.11517300 \\ \mathrm{~F} & 1.53921600 & 0.55458100 & -1.03524400 \\ \mathrm{~F} & 1.24341300 & -1.36066800 & -0.09476100\end{array}$

\section{$\mathrm{CF}_{3} \mathrm{SO}_{2} \mathrm{~N}$ (Triplet)}

\section{B3LYP/6-311++G(3df,3pd)}

Zero-point correction $=0.025655$ (Hartree/Particle)

Thermal correction to Energy $=0.033346$

Thermal correction to Enthalpy $=0.034290$

Thermal correction to Gibbs Free Energy $=-0.008503$

Sum of electronic and zero-point Energies $=-941.063248$

Sum of electronic and thermal Energies $=-941.055557$

Sum of electronic and thermal Enthalpies $=-941.054613$

Sum of electronic and thermal Free Energies $=-941.097406$

$\begin{array}{cccc}\mathrm{S} & -0.89907400 & 0.00000100 & -0.07527300 \\ \mathrm{O} & -1.31314800 & 1.25660900 & -0.61923300 \\ \mathrm{O} & -1.31312900 & -1.25661600 & -0.61922800 \\ \mathrm{~N} & -1.26327100 & 0.00000300 & 1.57535100 \\ \mathrm{C} & 0.98618600 & 0.00000400 & -0.00400900 \\ \mathrm{~F} & 1.43661300 & -0.00015300 & -1.24893500 \\ \mathrm{~F} & 1.41065700 & 1.08369200 & 0.63037000 \\ \mathrm{~F} & 1.41063900 & -1.08353900 & 0.63063700\end{array}$

\section{CBS-QB3}

Temperature $=298.150000$

Pressure $=1.000000$

$\mathrm{E}(\mathrm{ZPE})=0.025150$

$\mathrm{E}($ Thermal $)=0.032957$

$\mathrm{E}(\mathrm{SCF})=-937.956336$

$\mathrm{DE}(\mathrm{CBS})=-0.183953$

$\mathrm{DE}(\mathrm{CCSD})=-0.037232$

$\mathrm{DE}($ Empirical $)=-0.079786$

CBS-QB3 $(0 \mathrm{~K})=-939.888495$

CBS-QB3 Enthalpy= -939.879744

$\mathrm{DE}(\mathrm{MP} 2)=-1.679550$

$\mathrm{DE}(\mathrm{MP} 34)=-0.027664$

$\mathrm{DE}(\mathrm{Int})=0.050876$

CBS-QB3 Energy= -939.880688

CBS-QB3 Free Energy= -939.922948

$\begin{array}{lccc}\mathrm{S} & -0.89853100 & 0.00000100 & -0.07844000 \\ \mathrm{O} & -1.31456400 & 1.26480600 & -0.61989900 \\ \mathrm{O} & -1.31454300 & -1.26480900 & -0.61990100 \\ \mathrm{~N} & -1.25543500 & -0.00000200 & 1.58521000 \\ \mathrm{C} & 0.98581200 & 0.00000500 & -0.00514800 \\ \mathrm{~F} & 1.43894500 & -0.00019600 & -1.25283300 \\ \mathrm{~F} & 1.40734600 & 1.08692600 & 0.63223900 \\ \mathrm{~F} & 1.40732300 & -1.08673100 & 0.63257900\end{array}$

\section{M06-2X}

Zero-point correction $=0.027139$ (Hartree/Particle)

Thermal correction to Energy $=0.034592$

Thermal correction to Enthalpy= 0.035536 
Thermal correction to Gibbs Free Energy $=-0.006715$

Sum of electronic and zero-point Energies $=-940.847093$

Sum of electronic and thermal Energies $=-940.839640$

Sum of electronic and thermal Enthalpies $=-940.838696$

Sum of electronic and thermal Free Energies $=-940.880948$

$\begin{array}{lccc}\mathrm{S} & -0.88681200 & -0.00000500 & -0.08245600 \\ \mathrm{O} & -1.30058300 & 1.25138400 & -0.60950100 \\ \mathrm{O} & -1.30057700 & -1.25144500 & -0.60938600 \\ \mathrm{~N} & -1.19993500 & 0.00007600 & 1.58470800 \\ \mathrm{C} & 0.96465300 & 0.00000000 & -0.01247000 \\ \mathrm{~F} & 1.42518500 & -0.00011500 & -1.24358000 \\ \mathrm{~F} & 1.37684600 & 1.07685100 & 0.62459300 \\ \mathrm{~F} & 1.37684800 & -1.07673200 & 0.62479400\end{array}$

\section{MPW1PW91}

Zero-point correction $=0.026492$ (Hartree/Particle)

Thermal correction to Energy $=0.034062$

Thermal correction to Enthalpy $=0.035007$

Thermal correction to Gibbs Free Energy= -0.007530

Sum of electronic and zero-point Energies $=-940.935813$

Sum of electronic and thermal Energies $=-940.928243$

Sum of electronic and thermal Enthalpies $=-940.927298$

Sum of electronic and thermal Free Energies $=-940.969835$

$\begin{array}{cccc}\mathrm{S} & -0.88966600 & 0.00000000 & -0.07632500 \\ \mathrm{O} & -1.30181500 & 1.25097300 & -0.61362800 \\ \mathrm{O} & -1.30179700 & -1.25098300 & -0.61361600 \\ \mathrm{~N} & -1.24254500 & 0.00000700 & 1.56955000 \\ \mathrm{C} & 0.97486900 & 0.00000400 & -0.00518600 \\ \mathrm{~F} & 1.42215100 & -0.00013300 & -1.24265600 \\ \mathrm{~F} & 1.39516300 & 1.07726600 & 0.62584600 \\ \mathrm{~F} & 1.39514800 & -1.07713300 & 0.62607800\end{array}$

$\mathrm{FSO}_{2} \mathrm{~N}($ Singlet $)$

B3LYP/6-311++G(3df,3pd)

Zero-point correction $=0.014575$ (Hartree/Particle)

Thermal correction to Energy $=0.019093$

Thermal correction to Enthalpy $=0.020037$

Thermal correction to Gibbs Free Energy $=-0.013243$

Sum of electronic and zero-point Energies $=-703.183966$

Sum of electronic and thermal Energies $=-703.179449$

Sum of electronic and thermal Enthalpies $=-703.178504$

Sum of electronic and thermal Free Energies $=-703.211784$

$\begin{array}{lccc}\mathrm{S} & -0.05645600 & -0.12699600 & -0.04486300 \\ \mathrm{O} & 1.05399200 & 0.06691000 & 0.92420200 \\ \mathrm{O} & -1.06660700 & -1.10721800 & 0.06314400 \\ \mathrm{~N} & 1.19529900 & -0.11748000 & -0.93440200 \\ \mathrm{~F} & -0.81809600 & 1.24186300 & -0.07112700\end{array}$




\section{CBS-QB3}

Temperature $=298.150000$

$\mathrm{E}(\mathrm{ZPE})=0.013891$

$\mathrm{E}(\mathrm{SCF})=-701.051620$

$\mathrm{DE}(\mathrm{CBS})=-0.117241$

$\mathrm{DE}(\mathrm{CCSD})=-0.032898$

$\mathrm{DE}($ Empirical $)=-0.050372$

CBS-QB3 $(0 \mathrm{~K})=-702.319087$

CBS-QB3 Enthalpy= -702.313492

$\mathrm{S}$

$\mathrm{O}$

$\mathrm{O}$

$\mathrm{N}$

F

$$
\begin{gathered}
-0.04434000 \\
1.05017900 \\
-1.04046400 \\
1.19107600 \\
-0.85620200
\end{gathered}
$$

Pressure $=1.000000$

$\mathrm{E}($ Thermal $)=0.018543$

$\mathrm{DE}(\mathrm{MP} 2)=-1.103021$

$\mathrm{DE}(\mathrm{MP} 34)=-0.012705$

$\mathrm{DE}(\mathrm{Int})=0.034878$

CBS-QB3 Energy= -702.314436

CBS-QB3 Free Energy= -702.347030

\section{M06-2X}

Zero-point correction $=0.015522$ (Hartree/Particle)

Thermal correction to Energy $=0.019853$

Thermal correction to Enthalpy $=0.020798$

Thermal correction to Gibbs Free Energy $=-0.012131$

Sum of electronic and zero-point Energies $=-703.039082$

Sum of electronic and thermal Energies $=-703.034750$

Sum of electronic and thermal Enthalpies $=-703.033806$

Sum of electronic and thermal Free Energies $=-703.066735$

$\begin{array}{lccc}\mathrm{S} & -0.07318400 & -0.12299100 & -0.05513100 \\ \mathrm{O} & 1.06768100 & 0.03769500 & 0.87923000 \\ \mathrm{O} & -1.09214300 & -1.07953400 & 0.06079300 \\ \mathrm{~N} & 1.21119500 & -0.12541600 & -0.87715300 \\ \mathrm{~F} & -0.79019100 & 1.24227500 & -0.05533500\end{array}$

\section{MPW1PW91}

Zero-point correction $=0.015108$ (Hartree/Particle)

Thermal correction to Energy $=0.019535$

Thermal correction to Enthalpy $=0.020479$

Thermal correction to Gibbs Free Energy $=-0.012623$

Sum of electronic and zero-point Energies $=-703.108056$

Sum of electronic and thermal Energies $=-703.103628$

Sum of electronic and thermal Enthalpies $=-703.102684$

Sum of electronic and thermal Free Energies $=-703.135786$

$\begin{array}{lccc}\mathrm{S} & -0.06444700 & -0.12417400 & -0.04910600 \\ \mathrm{O} & 1.06006400 & 0.05143400 & 0.89990800 \\ \mathrm{O} & -1.07911200 & -1.08917200 & 0.06265900 \\ \mathrm{~N} & 1.19788100 & -0.12398500 & -0.90620100 \\ \mathrm{~F} & -0.80018200 & 1.23962100 & -0.06349300\end{array}$

$\mathrm{FSO}_{2} \mathrm{~N}($ Triplet) 
B3LYP/6-311++G(3df,3pd)

Zero-point correction $=0.014076$ (Hartree/Particle)

Thermal correction to Energy $=0.018693$

Thermal correction to Enthalpy $=0.019637$

Thermal correction to Gibbs Free Energy $=-0.014902$

Sum of electronic and zero-point Energies $=-703.215561$

Sum of electronic and thermal Energies $=-703.210944$

Sum of electronic and thermal Enthalpies $=-703.210000$

Sum of electronic and thermal Free Energies= -703.244539

$\begin{array}{lrrc}\mathrm{S} & 0.00004500 & -0.13385000 & -0.03106300 \\ \mathrm{O} & -1.24966100 & -0.68598700 & -0.41535100 \\ \mathrm{O} & 1.25011600 & -0.68515100 & -0.41536300 \\ \mathrm{~N} & -0.00002600 & 0.10370900 & 1.62256700 \\ \mathrm{~F} & -0.00046400 & 1.37608200 & -0.46836100\end{array}$

\section{CBS-QB3}

Temperature $=298.150000$

Pressure $=1.000000$

$\mathrm{E}(\mathrm{ZPE})=0.013408$

$\mathrm{E}(\mathrm{SCF})=-701.146278$

$\mathrm{E}($ Thermal $)=0.018163$

$\mathrm{DE}(\mathrm{CBS})=-0.113288$

$\mathrm{DE}(\mathrm{MP} 2)=-1.034959$

$\mathrm{DE}(\mathrm{CCSD})=-0.025641$

$\mathrm{DE}(\mathrm{MP} 34)=-0.018397$

$\mathrm{DE}($ Empirical $)=-0.048369$

CBS-QB3 $(0 \mathrm{~K})=-702.340923$

CBS-QB3 Enthalpy= -702.335224

$\mathrm{DE}(\mathrm{Int})=0.032600$

$\begin{array}{lrrr}\mathrm{S} & 0.14561400 & 0.00000000 & -0.03079800 \\ \mathrm{O} & 0.69184400 & -1.25825500 & -0.41582400 \\ \mathrm{O} & 0.69183900 & 1.25825700 & -0.41582500 \\ \mathrm{~N} & -0.11354000 & 0.00000000 & 1.62982400 \\ \mathrm{~F} & -1.40050100 & -0.00000200 & -0.47364400\end{array}$

\section{M06-2X}

Zero-point correction $=0.015003$ (Hartree/Particle)

Thermal correction to Energy $=0.019463$

Thermal correction to Enthalpy $=0.020407$

Thermal correction to Gibbs Free Energy= -0.013848

Sum of electronic and zero-point Energies $=-703.071447$

Sum of electronic and thermal Energies $=-703.066987$

Sum of electronic and thermal Enthalpies $=-703.066042$

Sum of electronic and thermal Free Energies $=-703.100297$

$\begin{array}{lrcc}\mathrm{S} & 0.00000000 & -0.13895100 & -0.01074700 \\ \mathrm{O} & -1.24301400 & -0.74931500 & -0.26680900 \\ \mathrm{O} & 1.24301100 & -0.74932200 & -0.26680800 \\ \mathrm{~N} & 0.00000000 & 0.44091600 & 1.56501800 \\ \mathrm{~F} & 0.00000400 & 1.23621100 & -0.72380400\end{array}$

\section{MPW1PW91}

Zero-point correction $=0.014600$ (Hartree/Particle $)$ 
Thermal correction to Energy $=0.019135$

Thermal correction to Enthalpy= 0.020079

Thermal correction to Gibbs Free Energy $=-0.014305$

Sum of electronic and zero-point Energies $=-703.143337$

Sum of electronic and thermal Energies $=-703.138802$

Sum of electronic and thermal Enthalpies $=-703.137858$

Sum of electronic and thermal Free Energies $=-703.172242$

$\begin{array}{crcc}\mathrm{S} & 0.00000000 & -0.13343300 & -0.02679400 \\ \mathrm{O} & -1.24382100 & -0.70133300 & -0.37680600 \\ \mathrm{O} & 1.24381800 & -0.70133800 & -0.37680700 \\ \mathrm{~N} & 0.00000100 & 0.18858000 & 1.60687800 \\ \mathrm{~F} & 0.00000200 & 1.33735800 & -0.53228200\end{array}$

\section{$\mathrm{CH}_{3} \mathrm{OSO}_{2} \mathrm{~N}$ (Singlet) \\ B3LYP/6-311++G(3df,3pd)}

Zero-point correction $=0.054787$ (Hartree/Particle)

Thermal correction to Energy $=0.061304$

Thermal correction to Enthalpy $=0.062248$

Thermal correction to Gibbs Free Energy $=0.023955$

Sum of electronic and zero-point Energies $=-718.442187$

Sum of electronic and thermal Energies $=-718.435670$

Sum of electronic and thermal Enthalpies $=-718.434726$

Sum of electronic and thermal Free Energies $=-718.473019$

$\begin{array}{lrrr}\mathrm{C} & -2.19514600 & -0.19705400 & 0.09221400 \\ \mathrm{H} & -2.89380700 & -0.96901500 & -0.21100700 \\ \mathrm{H} & -2.37041600 & 0.72415000 & -0.45403600 \\ \mathrm{H} & -2.25777300 & -0.02489700 & 1.16357100 \\ \mathrm{O} & -0.88882900 & -0.74006200 & -0.24922200 \\ \mathrm{~S} & 0.40032700 & 0.14371200 & -0.07359400 \\ \mathrm{O} & 0.00546300 & 1.51090200 & -0.00426000 \\ \mathrm{O} & 1.27055200 & -0.46659600 & 0.98632900 \\ \mathrm{~N} & 1.59859200 & -0.46874900 & -0.81958300\end{array}$

\section{CBS-QB3}

Temperature $=298.150000$

$\mathrm{E}(\mathrm{ZPE})=0.053643$

$\mathrm{E}(\mathrm{SCF})=-716.099305$

$\mathrm{DE}(\mathrm{CBS})=-0.129414$

$\mathrm{DE}(\mathrm{CCSD})=-0.038751$

$\mathrm{DE}($ Empirical $)=-0.057984$

CBS-QB3 $(0 \mathrm{~K})=-717.519226$

CBS-QB3 Enthalpy= -717.511625

$$
\begin{aligned}
& \text { Pressure }=1.000000 \\
& E(\text { Thermal })=0.060300 \\
& D E(M P 2)=-1.258917 \\
& D E(M P 34)=-0.029165 \\
& D E(\text { Int })=0.040667
\end{aligned}
$$

CBS-QB3 Energy= -717.512569

CBS-QB3 Free Energy= -717.550231
$-0.19169000$
0.09641200
$-0.92095000$
$-0.28033500$
0.77833100
$-0.37391100$
$-0.10983100$
1.18075400
$-0.73870400$
$-0.27241000$ 


$\begin{array}{lrrr}\mathrm{S} & 0.40989400 & 0.14473700 & -0.07096700 \\ \mathrm{O} & 0.01787900 & 1.51908100 & -0.00417900 \\ \mathrm{O} & 1.25231200 & -0.48053700 & 1.01175400 \\ \mathrm{~N} & 1.60065700 & -0.47313100 & -0.83583000\end{array}$

\section{M06-2X}

Zero-point correction $=0.056337$ (Hartree/Particle)

Thermal correction to Energy= 0.062534

Thermal correction to Enthalpy $=0.063479$

Thermal correction to Gibbs Free Energy $=0.026045$

Sum of electronic and zero-point Energies $=-718.280802$

Sum of electronic and thermal Energies $=-718.274605$

Sum of electronic and thermal Enthalpies $=-718.273661$

Sum of electronic and thermal Free Energies $=-718.311095$

$\begin{array}{lrrr}\mathrm{C} & -2.16921500 & -0.21455800 & 0.10064800 \\ \mathrm{H} & -2.86603500 & -1.00945900 & -0.13640000 \\ \mathrm{H} & -2.37546100 & 0.67463900 & -0.48572500 \\ \mathrm{H} & -2.19716100 & 0.01589000 & 1.16187500 \\ \mathrm{O} & -0.87426700 & -0.74762100 & -0.25427400 \\ \mathrm{~S} & 0.38473700 & 0.14949600 & -0.08484900 \\ \mathrm{O} & -0.03677400 & 1.49810600 & 0.00472700 \\ \mathrm{O} & 1.28442900 & -0.45796300 & 0.94599300 \\ \mathrm{~N} & 1.61586400 & -0.44654800 & -0.76537400\end{array}$

\section{MPW1PW91}

Zero-point correction $=0.055645$ (Hartree/Particle)

Thermal correction to Energy $=0.062058$

Thermal correction to Enthalpy $=0.063002$

Thermal correction to Gibbs Free Energy $=0.024920$

Sum of electronic and zero-point Energies $=-718.358877$

Sum of electronic and thermal Energies $=-718.352464$

Sum of electronic and thermal Enthalpies $=-718.351520$

Sum of electronic and thermal Free Energies= -718.389602

$\begin{array}{lrrr}\mathrm{C} & -2.17286800 & -0.20664800 & 0.09945800 \\ \mathrm{H} & -2.87854100 & -0.96249800 & -0.22679300 \\ \mathrm{H} & -2.34694600 & 0.73359900 & -0.41395000 \\ \mathrm{H} & -2.23528500 & -0.06963400 & 1.17597800 \\ \mathrm{O} & -0.88032700 & -0.73729800 & -0.25823300 \\ \mathrm{~S} & 0.39131900 & 0.14685700 & -0.07870300 \\ \mathrm{O} & -0.01017600 & 1.50402900 & -0.00133400 \\ \mathrm{O} & 1.27004500 & -0.46380700 & 0.96630700 \\ \mathrm{~N} & 1.60007700 & -0.46209700 & -0.78952200\end{array}$

\section{$\mathrm{CH}_{3} \mathrm{OSO}_{2} \mathrm{~N}$ (Triplet)}

\section{B3LYP/6-311++G(3df,3pd)}

Zero-point correction $=0.054251$ (Hartree/Particle)

Thermal correction to Energy $=0.060906$

Thermal correction to Enthalpy $=0.061850$ 
Thermal correction to Gibbs Free Energy $=0.022161$

Sum of electronic and zero-point Energies $=-718.473069$

Sum of electronic and thermal Energies $=-718.466414$

Sum of electronic and thermal Enthalpies $=-718.465470$

Sum of electronic and thermal Free Energies $=-718.505159$

$\begin{array}{cccc}\mathrm{C} & 2.13994700 & -0.03278700 & -0.00048400 \\ \mathrm{H} & 2.89335100 & -0.75113900 & -0.30464800 \\ \mathrm{H} & 2.30379800 & 0.27478200 & 1.02927900 \\ \mathrm{H} & 2.13165800 & 0.82530400 & -0.66502600 \\ \mathrm{O} & 0.88043500 & -0.75282200 & -0.10340000 \\ \mathrm{~S} & -0.48670400 & 0.03614400 & -0.06447000 \\ \mathrm{O} & -0.31391500 & 1.35495900 & -0.59260600 \\ \mathrm{O} & -1.48081700 & -0.85423200 & -0.56276100 \\ \mathrm{~N} & -0.72383500 & 0.18374700 & 1.57784900\end{array}$

\section{CBS-QB3}

Temperature $=298.150000$

Pressure $=1.000000$

$\mathrm{E}(\mathrm{ZPE})=0.053229$

$\mathrm{E}(\mathrm{SCF})=-716.192466$

$\mathrm{DE}(\mathrm{CBS})=-0.125456$

$\mathrm{DE}(\mathrm{CCSD})=-0.032071$

$\mathrm{DE}($ Empirical $)=-0.056052$

CBS-QB3 $(0 \mathrm{~K})=-717.540334$

CBS-QB3 Enthalpy= -717.532649

$\mathrm{E}($ Thermal $)=0.059969$

$\mathrm{DE}(\mathrm{MP} 2)=-1.189320$

$\mathrm{DE}(\mathrm{MP} 34)=-0.036563$

$\mathrm{DE}(\mathrm{Int})=0.038366$

CBS-QB3 Energy= -717.533593

CBS-QB3 Free Energy= -717.572385

$\begin{array}{cccc}\mathrm{C} & 2.14670800 & -0.03219300 & -0.00447900 \\ \mathrm{H} & 2.89631900 & -0.72515900 & -0.37862100 \\ \mathrm{H} & 2.34469800 & 0.21352400 & 1.03892200 \\ \mathrm{H} & 2.10945100 & 0.86663300 & -0.61670800 \\ \mathrm{O} & 0.88858000 & -0.76021200 & -0.10703100 \\ \mathrm{~S} & -0.49405600 & 0.04065400 & -0.06474200 \\ \mathrm{O} & -0.30949000 & 1.37205600 & -0.57264100 \\ \mathrm{O} & -1.48472300 & -0.85096900 & -0.58255200 \\ \mathrm{~N} & -0.72582200 & 0.15724300 & 1.58813500\end{array}$

M06-2X

Zero-point correction $=0.055970$ (Hartree/Particle $)$

Thermal correction to Energy $=0.062233$

Thermal correction to Enthalpy $=0.063178$

Thermal correction to Gibbs Free Energy $=0.024723$

Sum of electronic and zero-point Energies $=-718.311441$

Sum of electronic and thermal Energies $=-718.305178$

Sum of electronic and thermal Enthalpies $=-718.304233$

Sum of electronic and thermal Free Energies $=-718.342688$

$\begin{array}{cccc}\mathrm{C} & 2.09171700 & -0.04411100 & -0.00884000 \\ \mathrm{H} & 2.87043700 & -0.78663000 & -0.13455000 \\ \mathrm{H} & 2.14727000 & 0.39738200 & 0.98392400 \\ \mathrm{H} & 2.15739600 & 0.72421300 & -0.77246400\end{array}$




$\begin{array}{lccc}\mathrm{O} & 0.85383700 & -0.77126500 & -0.15630000 \\ \mathrm{~S} & -0.48117400 & 0.03463500 & -0.07121600 \\ \mathrm{O} & -0.30030200 & 1.33770000 & -0.60635000 \\ \mathrm{O} & -1.51713000 & -0.82808200 & -0.49499800 \\ \mathrm{~N} & -0.61683600 & 0.20981500 & 1.59668200\end{array}$

\section{MPW1PW91}

Zero-point correction $=0.055111$ (Hartree/Particle)

Thermal correction to Energy $=0.061656$

Thermal correction to Enthalpy $=0.062600$

Thermal correction to Gibbs Free Energy $=0.023194$

Sum of electronic and zero-point Energies $=-718.392959$

Sum of electronic and thermal Energies $=-718.386415$

Sum of electronic and thermal Enthalpies $=-718.385470$

Sum of electronic and thermal Free Energies $=-718.424877$

$\begin{array}{lccc}\mathrm{C} & 2.11399700 & -0.03692600 & -0.00087700 \\ \mathrm{H} & 2.87412100 & -0.74232200 & -0.31795300 \\ \mathrm{H} & 2.28188400 & 0.25682300 & 1.03245100 \\ \mathrm{H} & 2.10022700 & 0.83226800 & -0.65121700 \\ \mathrm{O} & 0.86909900 & -0.75505100 & -0.10987400 \\ \mathrm{~S} & -0.48143200 & 0.03477200 & -0.06676700 \\ \mathrm{O} & -0.30852000 & 1.34000200 & -0.60666200 \\ \mathrm{O} & -1.48284600 & -0.85130500 & -0.53403700 \\ \mathrm{~N} & -0.69416700 & 0.20703700 & 1.57354900\end{array}$

\section{$\mathrm{PhSO}_{2} \mathrm{~N}($ Singlet $)$}

\section{B3LYP/6-311++G(3df,3pd)}

Zero-point correction $=0.102974$ (Hartree/Particle)

Thermal correction to Energy $=0.111319$

Thermal correction to Enthalpy $=0.112263$

Thermal correction to Gibbs Free Energy $=0.068966$

Sum of electronic and zero-point Energies $=-834.951446$

Sum of electronic and thermal Energies $=-834.943101$

Sum of electronic and thermal Enthalpies $=-834.942157$

Sum of electronic and thermal Free Energies $=-834.985455$

$\begin{array}{lrrr}\mathrm{C} & 2.42287900 & 1.12716300 & 0.05487700 \\ \mathrm{C} & 1.03766500 & 1.22785900 & 0.02756100 \\ \mathrm{C} & 0.28280400 & 0.06057600 & -0.03273300 \\ \mathrm{C} & 0.88009600 & -1.19831600 & -0.07468400 \\ \mathrm{C} & 2.26301100 & -1.28116200 & -0.04212900 \\ \mathrm{C} & 3.03242500 & -0.12158100 & 0.02155300 \\ \mathrm{H} & 3.02316400 & 2.02490400 & 0.10189100 \\ \mathrm{H} & 0.54437500 & 2.18755500 & 0.05316800 \\ \mathrm{H} & 0.26449700 & -2.08348400 & -0.13750900 \\ \mathrm{H} & 2.74179700 & -2.24986500 & -0.07187700 \\ \mathrm{H} & 4.11126500 & -0.19390800 & 0.04208500 \\ \mathrm{~S} & -1.47626400 & 0.16187600 & -0.07194500 \\ \mathrm{O} & -1.85331100 & 1.53978700 & -0.11099400\end{array}$




$\begin{array}{rrrr}\mathrm{O} & -2.04806800 & -0.79327000 & 0.96971600 \\ \mathrm{~N} & -2.19530200 & -1.01922800 & -0.77615300\end{array}$

\section{CBS-QB3}

Temperature $=298.150000$

Pressure $=1.000000$

$\mathrm{E}(\mathrm{ZPE})=0.101462$

$\mathrm{E}($ Thermal $)=0.109968$

$\mathrm{E}(\mathrm{SCF})=-831.783775$

$\mathrm{DE}(\mathrm{CBS})=-0.177103$

$\mathrm{DE}(\mathrm{CCSD})=-0.068374$

$\mathrm{DE}($ Empirical $)=-0.078323$

CBS-QB3 $(0 \mathrm{~K})=-833.775011$

CBS-QB3 Enthalpy= -833.765561

$\mathrm{DE}(\mathrm{MP} 2)=-1.789923$

$\mathrm{DE}(\mathrm{MP} 34)=-0.037193$

$\mathrm{DE}(\operatorname{Int})=0.058217$

CBS-QB3 Energy $=-833.766505$

CBS-QB3 Free Energy= -833.809231

$\begin{array}{lrrr}\mathrm{C} & 2.42793900 & 1.13069100 & 0.05550500 \\ \mathrm{C} & 1.03939900 & 1.23172600 & 0.03002000 \\ \mathrm{C} & 0.28430200 & 0.06113900 & -0.02931700 \\ \mathrm{C} & 0.88118500 & -1.20103300 & -0.07542700 \\ \mathrm{C} & 2.26745600 & -1.28376300 & -0.04451000 \\ \mathrm{C} & 3.03838100 & -0.12125300 & 0.02018600 \\ \mathrm{H} & 3.02992900 & 2.03020400 & 0.10271400 \\ \mathrm{H} & 0.54326200 & 2.19265100 & 0.05680800 \\ \mathrm{H} & 0.26246700 & -2.08652300 & -0.14057500 \\ \mathrm{H} & 2.74793700 & -2.25424600 & -0.07674400 \\ \mathrm{H} & 4.11966200 & -0.19385100 & 0.03945000 \\ \mathrm{~S} & -1.48369200 & 0.16077200 & -0.07068300 \\ \mathrm{O} & -1.85756700 & 1.54617200 & -0.11691200 \\ \mathrm{O} & -2.04675400 & -0.79740600 & 0.98670800 \\ \mathrm{~N} & -2.19451200 & -1.02225000 & -0.79255000\end{array}$

\section{M06-2X}

Zero-point correction $=0.104337$ (Hartree/Particle)

Thermal correction to Energy= 0.112469

Thermal correction to Enthalpy $=0.113413$

Thermal correction to Gibbs Free Energy $=0.070668$

Sum of electronic and zero-point Energies $=-834.738207$

Sum of electronic and thermal Energies $=-834.730076$

Sum of electronic and thermal Enthalpies $=-834.729131$

Sum of electronic and thermal Free Energies $=-834.771876$

$\begin{array}{lrrr}\mathrm{C} & 2.41939800 & 1.12055500 & 0.04268900 \\ \mathrm{C} & 1.03668600 & 1.23198400 & 0.01701200 \\ \mathrm{C} & 0.28241300 & 0.06973200 & -0.03325400 \\ \mathrm{C} & 0.86104900 & -1.19323500 & -0.06376800 \\ \mathrm{C} & 2.24076400 & -1.28628800 & -0.03172800 \\ \mathrm{C} & 3.01586200 & -0.13187000 & 0.01984100 \\ \mathrm{H} & 3.02776400 & 2.01257100 & 0.07995800 \\ \mathrm{H} & 0.54381400 & 2.19273200 & 0.03650500 \\ \mathrm{H} & 0.22930400 & -2.06904100 & -0.11506200 \\ \mathrm{H} & 2.71371700 & -2.25736100 & -0.05219100 \\ \mathrm{H} & 4.09364800 & -0.21225400 & 0.03992300\end{array}$




$\begin{array}{lrrr}\mathrm{S} & -1.46497000 & 0.17488200 & -0.07426700 \\ \mathrm{O} & -1.84518800 & 1.54321400 & -0.05881200 \\ \mathrm{O} & -2.02879800 & -0.83317800 & 0.91263000 \\ \mathrm{~N} & -2.18769600 & -1.00147400 & -0.76230700\end{array}$

\section{MPW1PW91}

Zero-point correction $=0.103970$ (Hartree/Particle)

Thermal correction to Energy $=0.112230$

Thermal correction to Enthalpy $=0.113174$

Thermal correction to Gibbs Free Energy $=0.070085$

Sum of electronic and zero-point Energies $=-834.840961$

Sum of electronic and thermal Energies $=-834.832701$

Sum of electronic and thermal Enthalpies $=-834.831757$

Sum of electronic and thermal Free Energies $=-834.874846$

$\begin{array}{lrrr}\mathrm{C} & 2.41330000 & 1.12200600 & 0.05767200 \\ \mathrm{C} & 1.03220900 & 1.22674000 & 0.02888700 \\ \mathrm{C} & 0.27826000 & 0.06407300 & -0.03416100 \\ \mathrm{C} & 0.86792800 & -1.19376400 & -0.07818600 \\ \mathrm{C} & 2.24659700 & -1.28057200 & -0.04370200 \\ \mathrm{C} & 3.01651000 & -0.12570600 & 0.02303100 \\ \mathrm{H} & 3.01693300 & 2.01738900 & 0.10678900 \\ \mathrm{H} & 0.53805900 & 2.18646200 & 0.05555400 \\ \mathrm{H} & 0.24479800 & -2.07402400 & -0.14483400 \\ \mathrm{H} & 2.72333900 & -2.25024500 & -0.07492800 \\ \mathrm{H} & 4.09516900 & -0.20133900 & 0.04490800 \\ \mathrm{~S} & -1.46529200 & 0.16636900 & -0.07508300 \\ \mathrm{O} & -1.84252700 & 1.53671700 & -0.11008500 \\ \mathrm{O} & -2.03529000 & -0.79574400 & 0.95176500 \\ \mathrm{~N} & -2.18284500 & -1.02065500 & -0.74869300\end{array}$

\section{$\mathrm{PhSO}_{2} \mathrm{~N}$ (Triplet)}

\section{B3LYP/6-311++G(3df,3pd)}

Zero-point correction $=0.102296$ (Hartree/Particle)

Thermal correction to Energy $=0.110842$

Thermal correction to Enthalpy $=0.111786$

Thermal correction to Gibbs Free Energy $=0.067059$

Sum of electronic and zero-point Energies $=-834.975107$

Sum of electronic and thermal Energies $=-834.966562$

Sum of electronic and thermal Enthalpies $=-834.965617$

Sum of electronic and thermal Free Energies $=-835.010344$

$\begin{array}{lccc}\mathrm{C} & -2.31697100 & 1.20892700 & -0.00085700 \\ \mathrm{C} & -0.93055500 & 1.21756300 & -0.02886300 \\ \mathrm{C} & -0.24938800 & 0.00009700 & -0.04771400 \\ \mathrm{C} & -0.93042900 & -1.21747500 & -0.02892700 \\ \mathrm{C} & -2.31683100 & -1.20900400 & -0.00086800 \\ \mathrm{C} & -3.00700900 & -0.00007000 & 0.01536100 \\ \mathrm{H} & -2.85932500 & 2.14399800 & 0.00501400 \\ \mathrm{H} & -0.37640200 & 2.14421100 & -0.05329700\end{array}$




$\begin{array}{lrrr}\mathrm{H} & -0.37619800 & -2.14407100 & -0.05350900 \\ \mathrm{H} & -2.85908700 & -2.14413100 & 0.00504200 \\ \mathrm{H} & -4.08829200 & -0.00013200 & 0.03779300 \\ \mathrm{~S} & 1.51471900 & 0.00009500 & -0.06697200 \\ \mathrm{O} & 1.99697100 & 1.25346300 & -0.58362700 \\ \mathrm{O} & 1.99698000 & -1.25260000 & -0.58529400 \\ \mathrm{~N} & 1.83989800 & -0.00121900 & 1.57615600\end{array}$

\section{CBS-QB3}

Temperature $=298.150000$

$\mathrm{E}(\mathrm{ZPE})=0.100955$

$\mathrm{E}(\mathrm{SCF})=-831.871380$

$\mathrm{DE}(\mathrm{CBS})=-0.172195$

$\mathrm{DE}(\mathrm{CCSD})=-0.062913$

$\mathrm{DE}($ Empirical $)=-0.074271$

CBS-QB3 $(0 \mathrm{~K})=-833.786438$

CBS-QB3 Enthalpy= -833.776842

$$
\begin{aligned}
& \text { Pressure }=1.000000 \\
& \mathrm{E}(\text { Thermal })=0.109607 \\
& \mathrm{DE}(\mathrm{MP} 2)=-1.713471 \\
& \mathrm{DE}(\mathrm{MP} 34)=-0.048023 \\
& \mathrm{DE}(\text { Int })=0.054860
\end{aligned}
$$

CBS-QB3 Energy $=-833.777786$

CBS-QB3 Free Energy= -833.821752

$\begin{array}{cc}1.21227100 & -0.00073400 \\ 1.22099100 & -0.02821100 \\ 0.00001800 & -0.04224400 \\ -1.22097100 & -0.02854100 \\ -1.21228000 & -0.00105400 \\ -0.00001100 & 0.01521700 \\ 2.14914900 & 0.00340200 \\ 2.14793000 & -0.05547400 \\ -2.14789800 & -0.05607100 \\ -2.14916700 & 0.00283300 \\ -0.00002300 & 0.03664200 \\ 0.00002600 & -0.06805800 \\ 1.26215700 & -0.58823400 \\ -1.26182500 & -0.58892100 \\ -0.00045100 & 1.58403500\end{array}$

\section{M06-2X}

Zero-point correction $=0.103720$ (Hartree/Particle $)$

Thermal correction to Energy $=0.112104$

Thermal correction to Enthalpy $=0.113048$

Thermal correction to Gibbs Free Energy $=0.068596$

Sum of electronic and zero-point Energies $=-834.759663$

Sum of electronic and thermal Energies $=-834.751279$

Sum of electronic and thermal Enthalpies $=-834.750335$

Sum of electronic and thermal Free Energies $=-834.794787$

$\begin{array}{lrrr}\mathrm{C} & -2.30558600 & 1.20756800 & 0.00092700 \\ \mathrm{C} & -0.92077100 & 1.21628500 & -0.03862100 \\ \mathrm{C} & -0.24857200 & 0.00027100 & -0.06083500 \\ \mathrm{C} & -0.92040600 & -1.21600200 & -0.03873100 \\ \mathrm{C} & -2.30518500 & -1.20776600 & 0.00096600\end{array}$




$\begin{array}{lrrr}\mathrm{C} & -2.99217800 & -0.00019700 & 0.02274100 \\ \mathrm{H} & -2.84895400 & 2.14125100 & 0.01134600 \\ \mathrm{H} & -0.36025600 & 2.13970600 & -0.06775600 \\ \mathrm{H} & -0.35964600 & -2.13927300 & -0.06814500 \\ \mathrm{H} & -2.84824700 & -2.14162600 & 0.01147400 \\ \mathrm{H} & -4.07268700 & -0.00037600 & 0.05357400 \\ \mathrm{~S} & 1.50613000 & 0.00027200 & -0.07470200 \\ \mathrm{O} & 1.98895700 & 1.24961900 & -0.57042500 \\ \mathrm{O} & 1.98890400 & -1.24698800 & -0.57571700 \\ \mathrm{~N} & 1.81785900 & -0.00371900 & 1.58645700\end{array}$

\section{MPW1PW91}

Zero-point correction $=0.103258$ (Hartree/Particle)

Thermal correction to Energy $=0.111737$

Thermal correction to Enthalpy= 0.112681

Thermal correction to Gibbs Free Energy $=0.068091$

Sum of electronic and zero-point Energies $=-834.866754$

Sum of electronic and thermal Energies $=-834.858274$

Sum of electronic and thermal Enthalpies $=-834.857330$

Sum of electronic and thermal Free Energies $=-834.901920$

$\begin{array}{lrrc}\mathrm{C} & -2.30464600 & 1.20604200 & -0.00058000 \\ \mathrm{C} & -0.92179700 & 1.21461900 & -0.03212400 \\ \mathrm{C} & -0.24488700 & 0.00011400 & -0.05183800 \\ \mathrm{C} & -0.92164800 & -1.21451200 & -0.03218900 \\ \mathrm{C} & -2.30448100 & -1.20613100 & -0.00056900 \\ \mathrm{C} & -2.99192300 & -0.00008200 & 0.01760200 \\ \mathrm{H} & -2.84749900 & 2.14084500 & 0.00636500 \\ \mathrm{H} & -0.36429600 & 2.13968400 & -0.05874500 \\ \mathrm{H} & -0.36406600 & -2.13952200 & -0.05898200 \\ \mathrm{H} & -2.84721700 & -2.14100100 & 0.00643700 \\ \mathrm{H} & -4.07323500 & -0.00015500 & 0.04260400 \\ \mathrm{~S} & 1.50562200 & 0.00011200 & -0.06905100 \\ \mathrm{O} & 1.98662800 & 1.24815200 & -0.57656500 \\ \mathrm{O} & 1.98664400 & -1.24708600 & -0.57865300 \\ \mathrm{~N} & 1.82235400 & -0.00149400 & 1.57243800\end{array}$

